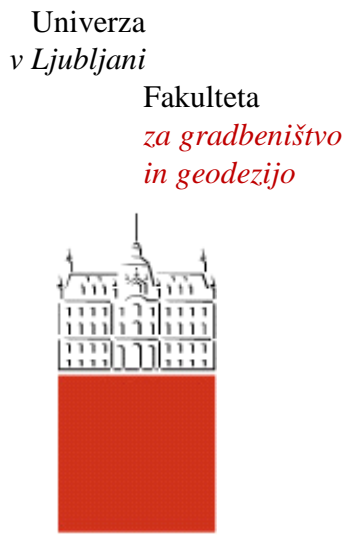

Jamova 2

1000 Ljubljana, Slovenija http://www3.fgg.uni-lj.si/

DRUGG - Digitalni repozitorij UL FGG http://drugg.fgg.uni-lj.si/

Ta članek je avtorjeva zadnja recenzirana različica, kot je bila sprejeta po opravljeni recenziji.

Prosimo, da se pri navajanju sklicujete na bibliografske podatke, kot je navedeno:

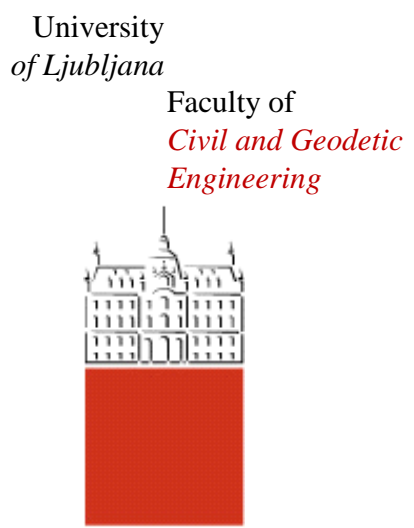

Jamova 2

SI - 1000 Ljubljana, Slovenia http://www3.fgg.uni-lj.si/en/

DRUGG - The Digital Repository http://drugg.fgg.uni-lj.si/

This version of the article is author's manuscript as accepted for publishing after the review process.

When citing, please refer to the publisher's bibliographic information as follows: 


\title{
Non-linear analysis of two-layer timber beams considering interlayer slip and uplift
}

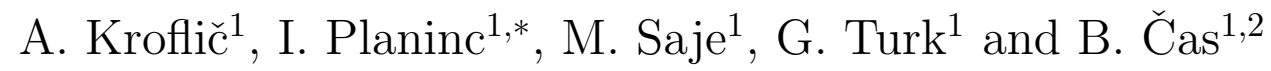 \\ 1 University of Ljubljana, Faculty of Civil and Geodetic Engineering, Jamova 2, \\ SI-1115 Ljubljana, Slovenia \\ ${ }^{2}$ Building and Civil Engineering Institute ZRMK, Dimičeva 12, SI-1000 \\ Ljubljana, Slovenia
}

\begin{abstract}
A new mathematical model and its finite element formulation for the non-linear analysis of mechanical behaviour of a two-layer timber planar beam is presented. A modified principle of virtual work is employed in formulating the finite element method. The basic unknowns are strains. The following assumptions are adopted in the mathematical model: materials are taken to be non-linear and can differ from layer to layer; interacting shear and normal contact tractions between layers are derived from the non-linear shear contact traction-slip and the non-linear normal contact traction-uplift characteristics of connectors; the geometrically linear and materially non-linear Bernoulli's beam theory is assumed for each layer. The formulation is found to be accurate, reliable and computationally effective. The suitability of the theory is validated by the comparison of the numerical solution and the experimental results of a full-scale laboratory tests on a simply supported beam. An excellent agreement between measured and calculated results is observed for all load levels. The further objective of the paper is the analysis of the effect of different normal contact traction-uplift constitutive relationships on the kinematic
\end{abstract}


and static quantities in a statically determined and undetermined structure. While the shear contact traction-slip constitutive relationship dictates the deformability of the composite beam and has a substantial infuence on most of the static and kinematic quantities of the composite beam, a variable normal contact traction-uplift constitutive relationship is in most cases neglegible.

Key words: non-linearity, composite beam, slip, uplift, timber, strain-based finite element

\section{Introduction}

Composite structure may be highly efficient structural form. If properly composed, they exhibit better bearing capacity and are easier to build. A particularly strong increase in research and application of composite structures has been observed in recent years in the rehabilitation of buildings and bridges.

The earliest theories dealing with composite planar beams were introduced in the middle of the last century after a number of experimental observations had confirmed the beneficial connected behaviour of layers. First mathematical theories of beams, composed of flexibly connected layers, were developed independently in Sweden [1], Soviet Union [2], Switzerland [3] and in the United States of America [4]. Most of subsequent theories consider linear elastic behaviour and small displacements (Girhammar and Gopu [5], Kroflič et al. [6], Ranzi et al. [7], see also Schnabl et al. [8,9]. A number of theories consider also non-linearity, as, e. g. Ayoub [10], who considered the material non-linearities,

* Corresponding author. E-mail address: iplaninc@fgg.uni-lj.si 
Betti and Gjelsvik [11], Čas et al. [12], Gattesco [13], Hirst and Yeo [14], Ranzi and Bradford [15], who took into account both material and geometric nonlinearities, Rassam and Goodman [16], Salari et al. [17], Seracino et al. [18], Thompson et al. [19]. Cas et al. [20] and Hozjan [21] seem to be the first to introduce a fully consistent materially and geometrically non-linear model of composite engineering beams.

The majority of the analysis procedures take into consideration solely an interlayer slip between the layers while neglecting uplift. The mathematical models which consider both slip and uplift at the contact were rarely proposed, e. g. in Adekola [22], Robinson and Naraine [23] who consider geometrically and materially linear behaviour whereas Gara et al. [24] consider bilinear constitutive law of materials.

The present paper proposes a finite element formulation for the materially nonlinear analysis of two-layer timber beams. Our formulation employs a modified principle of virtual work where the basic unknown functions are strains, i. e. deformation quantities. The Galerkin-type finite element formulation is employed as in Planinc et al. [25]. The present paper is focused on the effect of slip and uplift at the contact interface on mechanical behaviour of two-layer timber beams. The model considers the following assumptions: a composite structure, applied loading and deformations are planar; material of each layer is taken to be non-linear and homogeneous and can differ from layer to layer; interacting shear and normal contact tractions between the layers follow the non-linear shear traction-slip and normal traction-uplift characteristics of the connectors; the geometrically linear Bernoulli's beam theory is assumed for each layer; only a sufficiently small interlayer slip is assumed and the contact of layers where slip and uplift are realized, is modelled with an additional layer 
of small thickness. We note that it is straightforward to extend the present numerical model to a more general model of a multilayered composite beam.

The suitability of the present theoretical approach and its numerical solution for the analysis of a two-layer timber beam is verified by two characteristic cases. Firstly, we compare our numerical results to the analytical solution of an elastic two-layer beam (Kroflič et al. [6]). Then we compare numerical solutions of a simply supported non-linear beam modelled by different finite element meshes. Validation of the proposed procedure is performed by comparing the numerical solution with experimental results ([27], [30]). Finally, the influence of transverse stiffness of the connecting layers on static and kinematic quantities in the two-layer timber beam is examined.

\section{Basic equations of two-layer timber beam}

The static equilibrium of a two-layer timber beam is governed by the system of kinematic, equilibrium and constitutive equations with corresponding natural and essential boundary conditions for each layer. The compatibility of deformation between the layers is assured by proper constraining conditions $[20]$

\subsection{Kinematic, equilibrium and constitutive equations}

We assume planar, materially non-linear two-layer timber beam of length $L$. Equations of a more complex multi-layer timber or general composite beam can be derived similarly. 


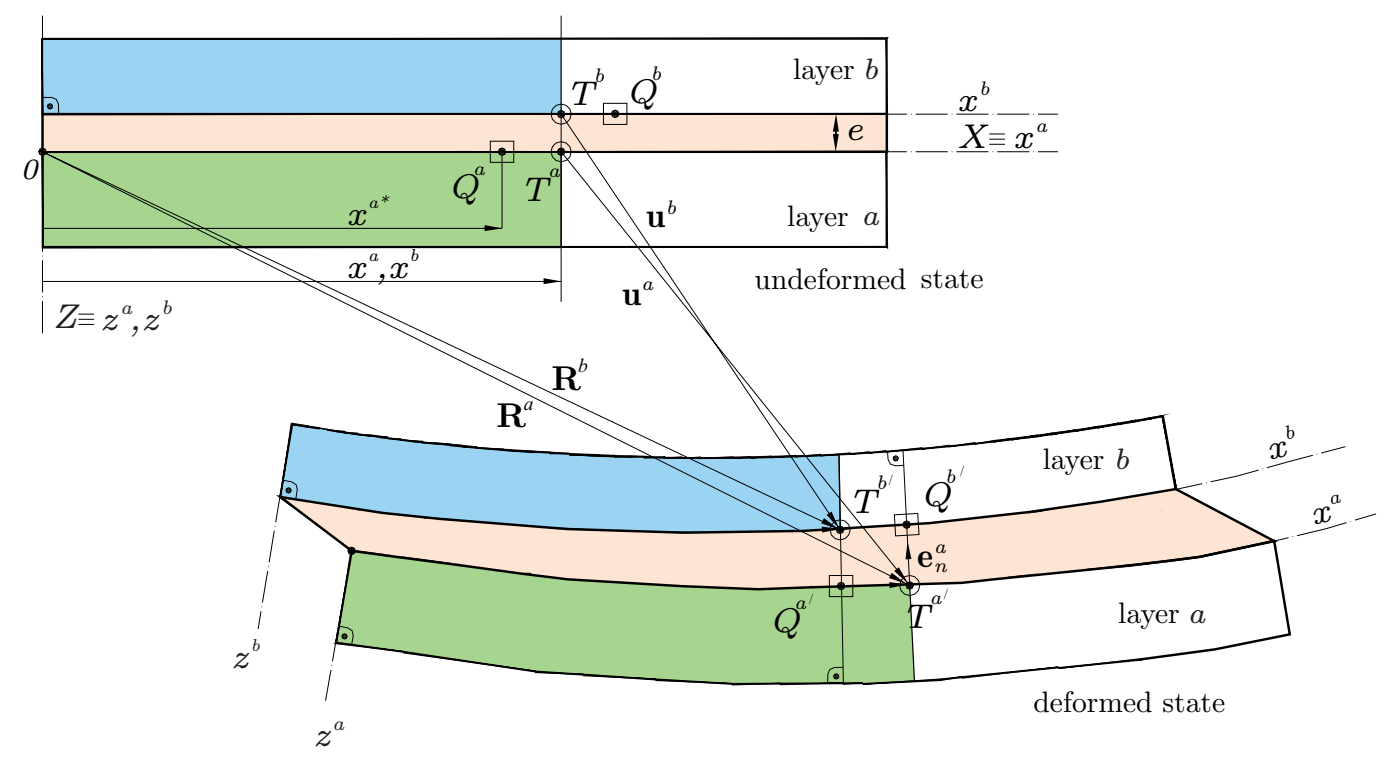

Fig. 1. Undeformed and deformed state of a two-layer timber beam.

We assume that deformations and rotations of each layer are small, so that the geometrically linear beam model is sufficient.

We consider deformations of a two-layer timber beam in a $(X, Z)$-plane of a fixed spatial Cartesian coordinate system $(X, Y, Z)$ with orthonormal base vectors $\mathbf{e}_{X}, \mathbf{e}_{Y}, \mathbf{e}_{Z}$, where $\mathbf{e}_{Y}=\mathbf{e}_{Z} \times \mathbf{e}_{X}$. We suppose the reference axis of an undeformed two-layer timber beam coincides with axis $X$. The deformed configurations of the axes of the two layers are defined by

$$
\begin{aligned}
& \mathbf{R}^{a}=x^{a} \mathbf{e}_{X}+\mathbf{u}^{a}=\left(x^{a}+u^{a}\right) \mathbf{e}_{X}+w^{a} \mathbf{e}_{Z} \\
& \mathbf{R}^{b}=x^{b} \mathbf{e}_{X}+\mathbf{u}^{b}=\left(x^{b}+u^{b}\right) \mathbf{e}_{X}+w^{b} \mathbf{e}_{Z}
\end{aligned}
$$

where $(\bullet)^{a}$ and $(\bullet)^{b}$ denote quantities related to layer $a$ and layer $b$, respectively. In Eqs. (1) functions $u^{a}$ and $w^{a}$ denote the $X$ and $Z$ components of the displacement vector of the axis of layer $a$; similarly, $u^{b}$ and $w^{b}$ belong to layer $b$. Material coordinates of both layers are denoted by $x^{a}, z^{a}$ and $x^{b}, z^{b}$ (Fig. 1). 
Bernoulli's hypothesis of planar cross-sections is assumed for each layer, i.e. the plane cross-section of each layer remains planar and perpendicular to the deformed axis. Hence the effect of shear strains is neglected. We can derive the following kinematic equations for the two layers:

$$
\begin{aligned}
u^{a \prime}-\varepsilon^{a}=0, & u^{b \prime}-\varepsilon^{b}=0, \\
w^{a \prime}+\varphi^{a}=0, & w^{b \prime}+\varphi^{b}=0, \\
\varphi^{a \prime}-\kappa^{a}=0, & \varphi^{b \prime}-\kappa^{b}=0,
\end{aligned}
$$

where $\varepsilon^{a}$ and $\varepsilon^{b}$ are extensional strains, $\kappa^{a}$ and $\kappa^{b}$ bending strains (curvatures), and $\varphi^{a}$ and $\varphi^{b}$ rotations of the axes of layers $a$ and $b$. The prime denotes the derivative with respect to $x^{a}$ or $x^{b}$.

Due to Bernoulli's hypothesis, the extensional strains, $D^{a}$ and $D^{b}$, of an arbitrary fibre in layers $a$ and $b$ are linear with respect to coordinate $z^{a}$ or $z^{b}$ :

$$
\begin{aligned}
D^{a} & =\varepsilon^{a}+z^{a} \kappa^{a}, \\
D^{b} & =\varepsilon^{b}+z^{b} \kappa^{b} .
\end{aligned}
$$

The equilibrium equations link axial forces $N^{a}, N^{b}$, shear forces $Q^{a}, Q^{b}$ and bending moments $M^{a}, M^{b}$ of two-layer timber beam with distributed loads $p_{x}^{a}, p_{x}^{b}, p_{z}^{a}, p_{z}^{b}, p_{t}^{a}, p_{n}^{a}, p_{t}^{b}, p_{n}^{b}, m_{y}^{a}, m_{y}^{b}$ :

$$
\begin{aligned}
N^{a \prime}+p_{x}^{a}+p_{t}^{a} & =0, & N^{b \prime}+p_{x}^{b}+p_{t}^{b} & =0, \\
Q^{a \prime}+p_{z}^{a}+p_{n}^{a} & =0, & Q^{b \prime}+p_{z}^{b}+p_{n}^{b} & =0, \\
M^{a \prime}-Q^{a}+m_{y}^{a} & =0, & M^{b \prime}-Q^{b}+m_{y}^{b} & =0,
\end{aligned}
$$

where $p_{t}^{a}, p_{t}^{b}$ and $p_{n}^{a}, p_{n}^{b}$ are components of the contact traction vector that acts in the contact plane. The next set of equations consists of constitutive equations. There are four equations which relate the equilibrium generalized 
internal forces $N^{a}, N^{b}, M^{a}, M^{b}$ to the constitutive generalized internal forces $N_{c}^{a}, N_{c}^{b}, M_{c}^{a}, M_{c}^{b}$ through extensional strains $D^{a}$ or $D^{b}$ :

$$
\begin{array}{r}
N^{a}=N_{c}^{a}=\int_{A^{a}} \sigma^{a}\left(D^{a}\right) d A, \\
N^{b}=N_{c}^{b}=\int_{A^{b}} \sigma^{b}\left(D^{b}\right) d A, \\
M^{a}=M_{c}^{a}=\int_{A^{a}} z^{a} \sigma^{a}\left(D^{a}\right) d A, \\
M^{b}=M_{c}^{b}=\int_{A^{b}} z^{b} \sigma^{b}\left(D^{b}\right) d A .
\end{array}
$$

The constitutive quantities, $N_{c}^{a}, N_{c}^{b}, M_{c}^{a}, M_{c}^{b}$, depend on chosen material models defined by the relationships $\sigma^{a}=\sigma^{a}\left(D^{a}\right)$ and $\sigma^{b}=\sigma^{b}\left(D^{b}\right)$, which need to be determined experimentally. The associated natural and essential boundary conditions are:

$$
x^{a}, x^{b}=0 \text { : }
$$

$$
\begin{aligned}
N^{a}(0)+S_{1}^{a}=0, & N^{b}(0)+S_{1}^{b}=0, \\
Q^{a}(0)+S_{2}^{a}=0, & Q^{b}(0)+S_{2}^{b}=0, \\
M^{a}(0)+S_{3}^{a}=0, & M^{b}(0)+S_{3}^{b}=0, \\
u^{a}(0)=u_{1}^{a}, & u^{b}(0)=u_{1}^{b}, \\
w^{a}(0)=u_{2}^{a}, & w^{b}(0)=u_{2}^{b}, \\
\varphi^{a}(0)=u_{3}^{a}, & \varphi^{b}(0)=u_{3}^{b}, \\
-N^{a}(L)+S_{4}^{a}=0, & \\
-Q^{a}(L)+S_{5}^{a}=0, & -N^{b}(L)+S_{4}^{b}=0, \\
-M^{a}(L)+S_{6}^{a}=0, & -M^{b}(L)+S_{6}^{b}=0,
\end{aligned}
$$




$$
\begin{array}{ll}
u^{a}(L)=u_{4}^{a}, & u^{b}(L)=u_{4}^{b}, \\
w^{a}(L)=u_{5}^{a}, & w^{b}(L)=u_{5}^{b}, \\
\varphi^{a}(L)=u_{6}^{a}, & \varphi^{b}(L)=u_{6}^{b} .
\end{array}
$$

In the above equations, $u_{i}^{a}$ and $u_{i}^{b}(i=1, \ldots, 6)$ denote the prescribed generalized boundary displacements, whereas $S_{i}^{a}$ and $S_{i}^{b}(i=1, \ldots, 6)$ are the prescribed generalized forces at the ends $x^{a}=x^{b}=0$ and $x^{a}=x^{b}=L$ of layers $a$ and $b$.

\subsection{Constraining equations}

Layers slip with respect to each other and may eventually separate during the deformation. If the layers are very stiff compared to the stiffness of connectors, or if they are not connected at all, a substantial slip and uplift may occur at the contact. If layers are nearly as stiff as the connectors or if one layer is much softer than the other, slip and uplift are small and can thus be attributed to a thin connecting layer.

For this reason, slip and uplift are in the present paper defined as an average slip and an average uplift over a thin connecting layer made of soft material, rather than slip and uplift over an actual contact interface (Fig. 2). Hence, the interaction between the layers $a$ and $b$ is achieved through the connecting layer of thickness $e$, thus being more a computational than a geometric property, yet depending on characteristics of layers and connectors [12]. The characteristics of layers must be found by a specially designed experiment. Once obtained, the generalized slip (or uplift) is used in a shear traction-slip or normal traction-uplift relationship. The shear traction-slip and normal traction-uplift 
relationships are assumed to be non-linear which is in accordance with experiments [26]. The introduction of thickness $e$ seems to be physically sound and adds an additional material parameter to the mathematical model.

The constraining conditions employed to assemble the layers into the composite beam (Fig. 1) require continuity of the possibly uplifted contact surface as:

$$
\mathbf{R}^{a}\left(T^{a^{\prime}}\right)-d^{a} \mathbf{e}_{n}^{a}=\mathbf{R}^{b}\left(Q^{b^{\prime}}\right)
$$

where $\mathbf{e}_{n}^{a}$ presents the unit vector perpendicular to the contact surface of layer $a$ at the point $T^{a^{\prime}}$. Coordinate $x^{b *}$ represents a material, undeformed coordinate of particle $Q^{b}$ of layer $b$ which, in the deformed state, gets in contact with particle $T^{a}$ of layer $a$ with coordinate $x^{a}$. Coordinates $z^{s a}$ and $z^{s b}$ present the vertical coordinate (distance) of points $T^{a}$ and $Q^{b}$ from the corresponding layer reference axis. After assuming small slips and performing the linearization, the componential form of Eq. (10) is written as

$$
\begin{array}{r}
x^{a}+u^{a}+e \varphi^{a}=x^{b *}+u^{b}, \\
w^{a}-d^{a}=w^{b},
\end{array}
$$

where $d^{a}=d+e$. The unknown function $d$ of $x^{a}$ stands for uplift at the contact between layers and can be calculated from Eq. (12) as

$$
d=w^{a}-w^{b}-e
$$

The slip which occurs between two points that coincide in the undeformed shape is denoted by $\Delta$. The relation between $\Delta$ and kinematic quantities can be derived from (11)

$$
\Delta=x^{b *}-x^{a}=u^{a}-u^{b}+e \varphi^{a} .
$$


The geometrical meaning of uplift $d$, and slip $\Delta$, is further described in Fig. 2. The stiffness of the contact depends on the connecting materials and the

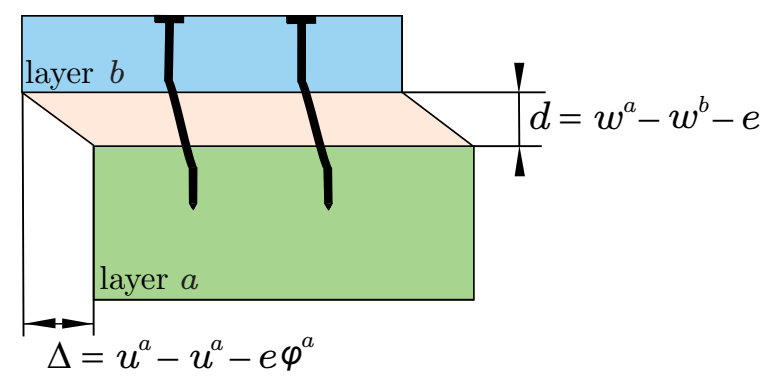

Fig. 2. Geometrical meaning of slip and uplift.

design details of the contact. Our constitutive contact equations are assumed to be defined in a general form as

$$
\begin{aligned}
& p_{t}^{a}=-p_{t}^{b}=F(\Delta), \\
& p_{n}^{a}=-p_{n}^{b}=G(d) .
\end{aligned}
$$

For a given geometry of the beam, external loadings and boundary conditions, Eqs. (2), (4-5), (11), (13-15) constitute a system of 23 equations for 23 unknown functions $u^{a}\left(x^{a}\right), u^{b}\left(x^{b}\right), w^{a}\left(x^{a}\right), w^{b}\left(x^{b}\right), \varphi^{a}\left(x^{a}\right), \varphi^{b}\left(x^{b}\right), \varepsilon^{a}\left(x^{a}\right), \varepsilon^{b}\left(x^{b}\right)$, $\kappa^{a}\left(x^{a}\right), \kappa^{b}\left(x^{b}\right), N^{a}\left(x^{a}\right), N^{b}\left(x^{b}\right), Q^{a}\left(x^{a}\right), Q^{b}\left(x^{b}\right), M^{a}\left(x^{a}\right), M^{b}\left(x^{b}\right), \Delta\left(x^{a}\right), d\left(x^{a}\right)$, $p_{t}^{a}\left(x^{a}\right), p_{t}^{b}\left(x^{b}\right), p_{n}^{a}\left(x^{a}\right), p_{n}^{b}\left(x^{b}\right)$ and $x^{b *}\left(x^{a}\right)$ with the corresponding natural and essential boundary conditions (6-9).

\section{The finite element formulation}

The analytical solution of the stress-strain state of a composite beam is possible only when material models of layers and contact are linear (Adekola [22], Kroflič [6], Robinson and Naraine [23]). Otherwise the system of equations can only be solved by a numerical method, e.g. the finite element method. 
The present numerical method employs the strain-based finite elements $[12,20]$. It is derived from the modified principle of virtual work, which makes it possible to introduce the strains in a natural way as the only unknowns of the system of equation, while the remaining unknowns are included only as boundary values in the functional. The modified principle of virtual work is (Čas et al. [20]): 


$$
\begin{aligned}
\delta W^{*}= & \delta W^{a *}+\delta W^{b *}= \\
& \int_{0}^{L}\left(\left(N_{c}^{a}-N^{a}\right) \delta \varepsilon^{a}+\left(M_{c}^{a}-M^{a}\right) \delta \kappa^{a}\right) d x^{a}+ \\
& +\left(u^{a}(L)-u^{a}(0)-\int_{0}^{L} \varepsilon^{a} d \xi\right) \delta N^{a}(0)+ \\
& +\left(w^{a}(L)-w^{a}(0)+\int_{0}^{L} \varphi^{a} d \xi\right) \delta Q^{a}(0)+ \\
& +\left(\varphi^{a}(L)-\varphi^{a}(0)-\int_{0}^{L} \kappa^{a} d \xi\right) \delta M^{a}(0)+ \\
& +\left(-S_{1}^{a}-N^{a}(0)\right) \delta u^{a}(0)+\left(-S_{2}^{a}-Q^{a}(0)\right) \delta w^{a}(0)+ \\
& +\left(-S_{3}^{a}-M^{a}(0)\right) \delta \varphi^{a}(0)+\left(-S_{4}^{a}+N^{a}(L)\right) \delta u^{a}(L)+ \\
& +\left(-S_{5}^{a}+Q^{a}(L)\right) \delta w^{a}(L)+\left(-S_{6}^{a}+M^{a}(L)\right) \delta \varphi^{a}(L)+ \\
& +\int_{0}^{L}\left(\left(N_{c}^{b}-N^{b}\right) \delta \varepsilon^{b}+\left(M_{c}^{b}-M^{b}\right) \delta \kappa^{b}\right) d x^{b}+ \\
& +\left(u^{b}(L)-u^{b}(0)-\int_{0}^{L} \varepsilon^{b} d \xi\right) \delta N^{b}(0)+ \\
& +\left(w^{b}(L)-w^{b}(0)+\int_{0}^{L} \varphi^{b} d \xi\right) \delta Q^{b}(0)+ \\
& +\left(\varphi^{b}(L)-\varphi^{b}(0)-\int_{0}^{L} \kappa^{b} d \xi\right) \delta M^{b}(0)+ \\
& +\left(-S_{1}^{b}-N^{b}(0)\right) \delta u^{b}(0)+\left(-S_{2}^{b}-Q^{b}(0)\right) \delta w^{b}(0)+ \\
& \left(-M^{b}(0)\right) \delta \varphi^{b}(0)+\left(-S_{4}^{b}+N^{b}(L)\right) \delta u^{b}(L)+ \\
& \left(-w^{b}(L)+\left(-S_{6}^{b}+M^{b}(L)\right) \delta \varphi^{b}(L)\right.
\end{aligned}
$$

The only unknown variables of the problem are strains $\varepsilon^{a}\left(x^{a}\right), \varepsilon^{b}\left(x^{b}\right), \kappa^{a}\left(x^{a}\right)$ and $\kappa^{b}\left(x^{b}\right)$, generalized boundary forces $N^{a}(0), Q^{a}(0), M^{a}(0), N^{b}(0), Q^{b}(0)$, $M^{b}(0)$, and boundary kinematic quantities $u^{a}(0), u^{a}(L), w^{a}(0), w^{a}(L), \varphi^{a}(0)$, $\varphi^{a}(L), u^{b}(0), u^{b}(L), w^{b}(0), w^{b}(L), \varphi^{b}(0)$ and $\varphi^{a}(L)$.

The extensional strain, $\varepsilon^{a}$, of layer $a$, the extensional strain, $\varepsilon^{b}$, of layer $b$ and 
the bending strains, $\kappa^{a}$ and $\kappa^{b}$, are interpolated by the Lagrangian polynomials $L_{m}(m=1,2, \ldots, M)$ of the same order $(M-1)$ :

$$
\begin{aligned}
\varepsilon^{a}\left(x^{a}\right) & =\sum_{m=1}^{M} L_{m}\left(x^{a}\right) \varepsilon_{m}^{a}, \\
\varepsilon^{b}\left(x^{b}\right) & =\sum_{m=1}^{M} L_{m}\left(x^{b}\right) \varepsilon_{m}^{b}, \\
\kappa^{a}\left(x^{a}\right) & =\sum_{m=1}^{M} L_{m}\left(x^{a}\right) \kappa_{m}^{a}, \\
\kappa^{b}\left(x^{b}\right) & =\sum_{m=1}^{M} L_{m}\left(x^{b}\right) \kappa_{m}^{b} .
\end{aligned}
$$

Interpolation points are taken to be equidistant. The scalar values $\varepsilon_{m}^{a}, \varepsilon_{m}^{b}, \kappa_{m}^{a}$ and $\kappa_{m}^{b}$ denote the nodal values of the extensional and bending strains. When the interpolated strains in Eqs. (17) are inserted into the modified principle of virtual work (16), we obtain the system of generalized equilibrium equations of a two-layer timber beam accounting for interlayer slip and uplift:

$$
\begin{aligned}
& g_{i}=\int_{0}^{L}\left(N_{c}^{a}-N^{a}\right) L_{i} d \xi=0, \quad i=1, \ldots, M \\
& g_{M+i}=\int_{0}^{L}\left(N_{c}^{b}-N^{b}\right) L_{i} d \xi=0, \quad i=1, \ldots, M \\
& g_{2 M+i}=\int_{0}^{L}\left(M_{c}^{a}-M^{a}\right) L_{i} d \xi=0, \quad i=1, \ldots, M \\
& g_{3 M+i}=\int_{0}^{L}\left(M_{c}^{b}-M^{b}\right) L_{i} d \xi=0, \quad i=1, \ldots, M \\
& g_{4 M+1}=u^{a}(L)-u^{a}(0)-\int_{0}^{L} \varepsilon^{a} d \xi=0, \\
& g_{4 M+2}=w^{a}(L)-w^{a}(0)-\int_{0}^{L} \varphi^{a} d \xi=0, \\
& g_{4 M+3}=\varphi^{a}(L)-\varphi^{a}(0)-\int_{0}^{L} \kappa^{a} d \xi=0, \\
& g_{4 M+4}=u^{b}(L)-u^{b}(0)-\int_{0}^{L} \varepsilon^{b} d \xi=0, \\
& g_{4 M+5}=w^{b}(L)-w^{b}(0)-\int_{0}^{L} \varphi^{b} d \xi=0, \\
& g_{4 M+6}=\varphi^{b}(L)-\varphi^{b}(0)-\int_{0}^{L} \kappa^{b} d \xi=0, \\
& g_{4 M+7}=-S_{1}^{a}-N^{a}(0)=0,
\end{aligned}
$$




$$
\begin{aligned}
& g_{4 M+8}=-S_{2}^{a}-Q^{a}(0)=0, \\
& g_{4 M+9}=-S_{3}^{a}-M^{a}(0)=0, \\
& g_{4 M+10}=-S_{1}^{b}-N^{b}(0)=0, \\
& g_{4 M+11}=-S_{2}^{b}-Q^{b}(0)=0, \\
& g_{4 M+12}=-S_{3}^{b}-M^{b}(0)=0, \\
& g_{4 M+13}=S_{4}^{a}-N^{a}(L)=0, \\
& g_{4 M+14}=S_{5}^{a}-Q^{a}(L)=0, \\
& g_{4 M+15}=S_{6}^{a}-M^{a}(L)=0, \\
& g_{4 M+16}=S_{4}^{b}-N^{b}(L)=0, \\
& g_{4 M+17}=S_{5}^{b}-Q^{b}(L)=0, \\
& g_{4 M+18}=S_{6}^{b}-M^{b}(L)=0 .
\end{aligned}
$$

The above non-linear algebraic system of generalized equilibrium equations of the two-layer timber beam constitutes a system of $4 M+18$ equations with as many so called primary unknowns. These consist of $4 M+6$ internal degrees of freedom, $\varepsilon_{m}^{a}, \varepsilon_{m}^{b}, \kappa_{m}^{a}, \kappa_{m}^{b}(m=1,2, \ldots, M), N^{a}(0), N^{b}(0), Q^{a}(0), Q^{b}(0), M^{a}(0)$, $M^{b}(0)$, and 12 external degrees of freedom, $u^{a}(0), u^{b}(0), w^{a}(0), w^{b}(0), \varphi^{a}(0)$, $\varphi^{b}(0), u^{a}(L), \quad u^{b}(L), w^{a}(L), w^{b}(L), \quad \varphi^{a}(L), \varphi^{b}(L)$, of a finite element. The secondary unknown functions $u^{a}, u^{b}, w^{a}, w^{b}, \varphi^{a}, \varphi^{b}, N^{a}, N^{b}, Q^{a}, Q^{b}, M^{a}$, $M^{b}, \Delta, d, p_{t}^{a}, p_{n}^{a}, p_{t}^{b}, p_{n}^{b}, x^{b *}$ when needed at a particular value of $x^{a}$ or $x^{b}$ in the above equations are obtained by the equations:

$$
\begin{aligned}
& u^{a}\left(x^{a}\right)=u^{a}(0)+\int_{0}^{x^{a}} \varepsilon^{a} d \xi, \\
& w^{a}\left(x^{a}\right)=w^{a}(0)+\int_{0}^{x^{a}} \varphi^{a} d \xi, \\
& \varphi^{a}\left(x^{a}\right)=\varphi^{a}(0)+\int_{0}^{x^{a}} \kappa^{a} d \xi, \\
& u^{b}\left(x^{b}\right)=u^{b}(0)+\int_{0}^{x^{b}} \varepsilon^{b} d \xi,
\end{aligned}
$$




$$
\begin{aligned}
& w^{b}\left(x^{b}\right)=w^{b}(0)+\int_{0}^{x^{b}} \varphi^{b} d \xi \\
& \varphi^{b}\left(x^{b}\right)=\varphi^{b}(0)+\int_{0}^{x^{b}} \kappa^{b} d \xi \\
& N^{a}\left(x^{a}\right)=N^{a}(0)-\int_{0}^{x^{a}}\left(p_{x}^{a}+p_{t}^{a}\right) d \xi \\
& Q^{a}\left(x^{a}\right)=Q^{a}(0)-\int_{0}^{x^{a}}\left(p_{z}^{a}+p_{n}^{a}\right) d \xi \\
& M^{a}\left(x^{a}\right)=M^{a}(0)+\int_{0}^{x^{a}}\left(Q^{a}-m_{y}^{a}\right) d \xi \\
& N^{b}\left(x^{b}\right)=N^{b}(0)-\int_{0}^{x^{b}}\left(p_{x}^{b}+p_{t}^{b}\right) d \xi \\
& Q^{b}\left(x^{b}\right)=Q^{b}(0)-\int_{0}^{x^{b}}\left(p_{z}^{b}+p_{n}^{b}\right) d \xi \\
& M^{b}\left(x^{b}\right)=M^{b}(0)+\int_{0}^{x^{b}}\left(Q^{b}-m_{y}^{b}\right) d \xi \\
& x^{b *}\left(x^{a}\right)=x^{a}+u^{a}-u^{b}+e \varphi^{a}, \\
& d\left(x^{a}\right)=w^{a}-w^{b}-e, \\
& \Delta\left(x^{a}\right)=u^{a}-u^{b}+e \varphi^{a}, \\
& p_{t}^{a}\left(x^{a}\right)=F(\Delta) \\
& p_{n}^{a}\left(x^{a}\right)=G(d), \\
& p_{t}^{b}\left(x^{b}\right)=-p_{t}^{a}\left(x^{a}\right), \\
& p_{n}^{b}\left(x^{b}\right)=-p_{n}^{a}\left(x^{a}\right) .
\end{aligned}
$$

The Newton-Raphson method is employed for the solution of this algebraic system of equations.

\section{Experimental work}

- Experiment A

In order to validate the numerical model, we compared our numerical results with experimentally obtained results of Planinc et al. [27], who performed several full-scale laboratory tests to study the mechanical properties 
of timber, contact parameters between connected timber elements and the deformability of simply supported timber composite beams. Only a brief review of the testing procedures and their results are presented in this paper, since the details of the experiment have already been presented in Planinc et al. [27] and the references therein. A simply supported, $3000 \mathrm{~mm}$ long composite timber beam with a span $L=2800 \mathrm{~mm}$ was studied. The beam was composed from two timber rafters of equal length and width, but different heights. They were connected to each other with standard nails 40/100. The axial distance between the nails was $60 \mathrm{~mm}$.

The deflections of the beam axis, slip along the contact and the following material characteristics of timber and the interface connection were measured in the experiment:

- compressive strength along the grain,

- tensile strength along the grain,

- shear stiffness and load bearing capacity of connectors,

- tensile drag characteristics of connectors.

Experiments of the timber compressive and tensile constitutive law, pullout strength tests for defining the tensile drag characteristics of connectors, and the shear flow-slip tests of the contact are fully described in [26].

The two-layer timber cross-section is presented in Fig. 3. In accordance with the EN 338 [28] classification, timber has been classified in strength class C24. The nails 40/100 were arranged in two parallel rows and uniformly distributed along the contact interface as seen in Fig. 3.

A $100 \mathrm{~mm}$ long cantilevers at the supports (Fig. 3) were neglected in mathematical modelling. The assumption appears to be reasonable due to small relative length and dead load of the cantilevers. The beam is subjected to dead load and loaded with a slowly increasing point force, $P$, at the 
cross-section

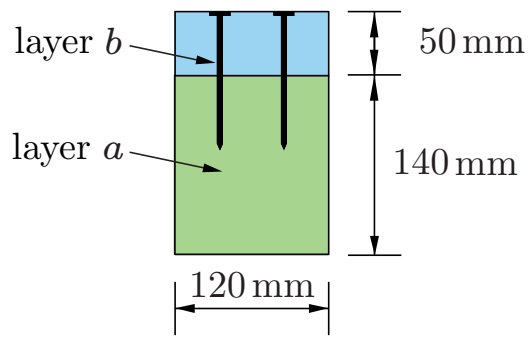

longitudinal arrangement of connectors

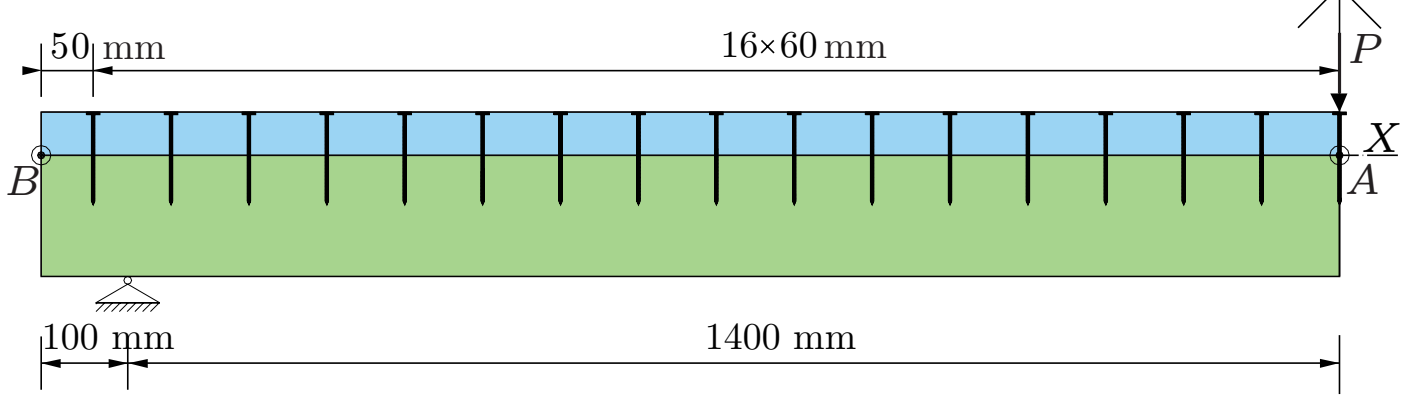

Fig. 3. Cross-section of the composite timber beam and the arrangement of connectors.

midspan.

Material parameters of the constitutive law of timber were according to [27] determined in a series of compressive and tensile tests on timber specimens. The constitutive law displayed in Fig. 4 was used in the numerical simulations. Based on experiments, the elastic modulus of timber was estimated as being $E_{t}=E_{c}=11500 \mathrm{~N} / \mathrm{mm}^{2}$ in tension and compression; the remaining material properties of timber were estimated as being: $D_{t y}=0.32 \%, D_{t u}=1.00 \%, D_{c y}=0.35 \%, D_{c u}=1.03 \%, E_{c h}=$ $0.1 E_{c}, E_{t h}=0.05 E_{t}$. Ultimate deformations needed to describe the timber constitutive law were determined according to Pischl [29].

In a parallel experiment, the shear traction-slip $\left(p_{t}^{a}-\Delta\right)$ and the normal traction-uplift $\left(p_{n}^{a}-d\right)$ relations for a nailed contact were obtained. These experiments are fully described in Čas [26]. There were three specimens chosen for the determination of each constitutive law. The dimensions and 


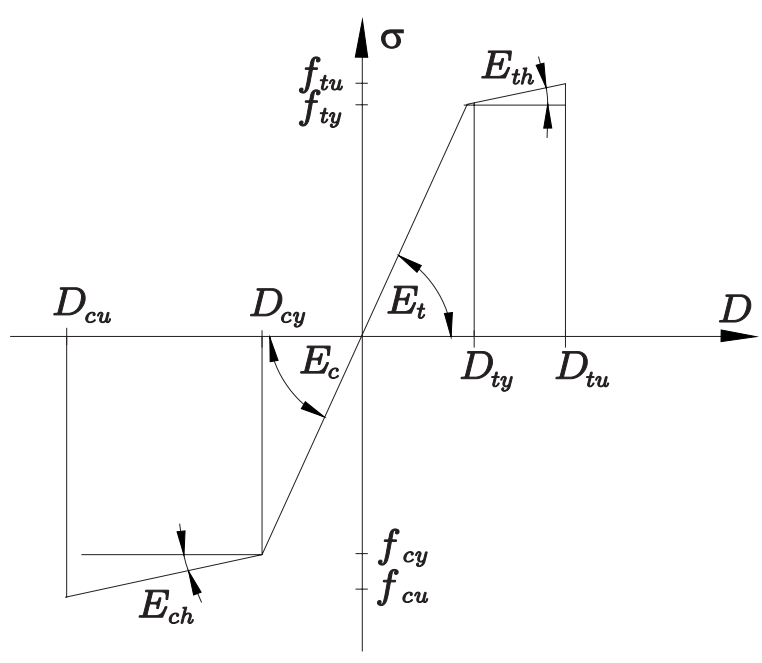

Fig. 4. Constitutive law of timber used in the analysis.

the arrangement of nails in the specimens are presented in Fig. 5 (specimens for the shear traction-slip relation) and in Fig. 6 (the ones for the normal traction-uplift relation). Both kinds of experiments were performed in the universal testing machine INSTRON 1345. It should also be noted that the arrangement of the nails in the test specimen was similar to the arrangement of the nails in the tested timber composite beams.

The computational contact constitutive law was determined by averaging the results of all measurements. The goal of the experiments was to gain the shear traction-slip and the normal traction-uplift relations for a single nail, so each averaged constitutive diagram was also adequately reduced with the number of nails used in experiments.

The experiments clearly showed that the relations between the shear contact traction and the related slip, as well as the normal contact traction and the related uplift between the layers are non-linear. Fig. 7 presents the shear traction-slip and the normal traction-uplift relations, as obtained experimentally and employed in the numerical simulations.

- Experiment B 


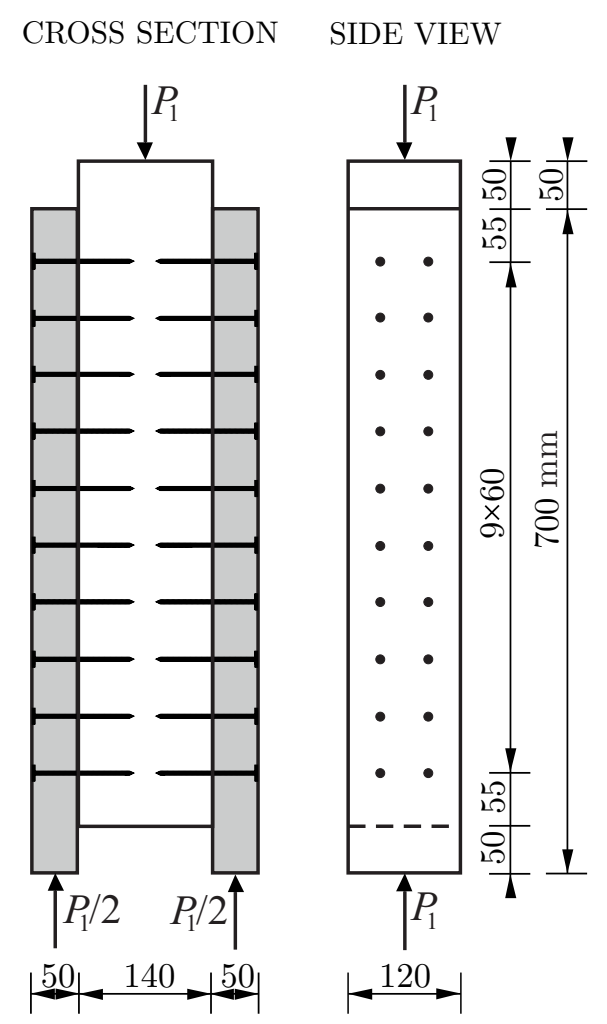

Fig. 5. Geometrical data of shear specimens and the arrangement of nails.

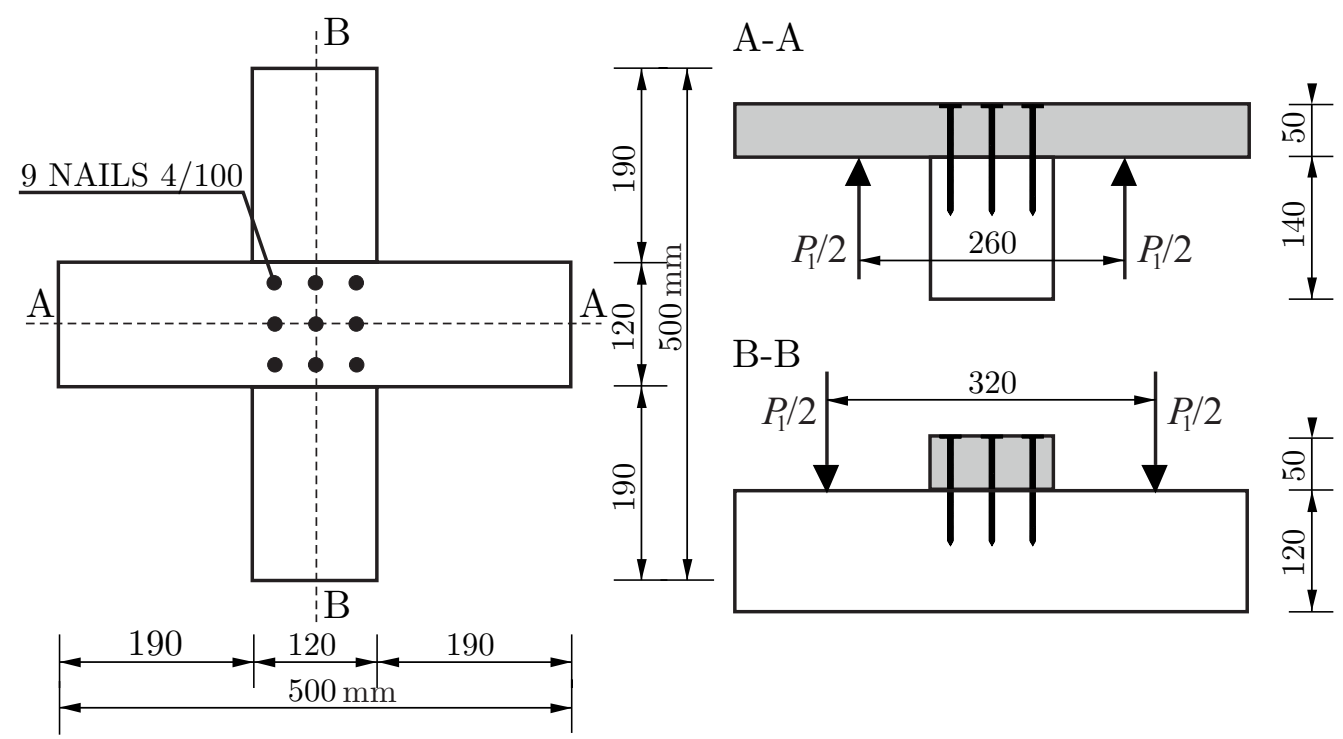

Fig. 6. Geometrical data of uplift specimens and the arrangement of nails.

We also compare our numerical results with experimentally obtained results of McCutcheon [30] who performed several full-scale laboratory tests on T-beam 
(a) shear traction-slip relationship

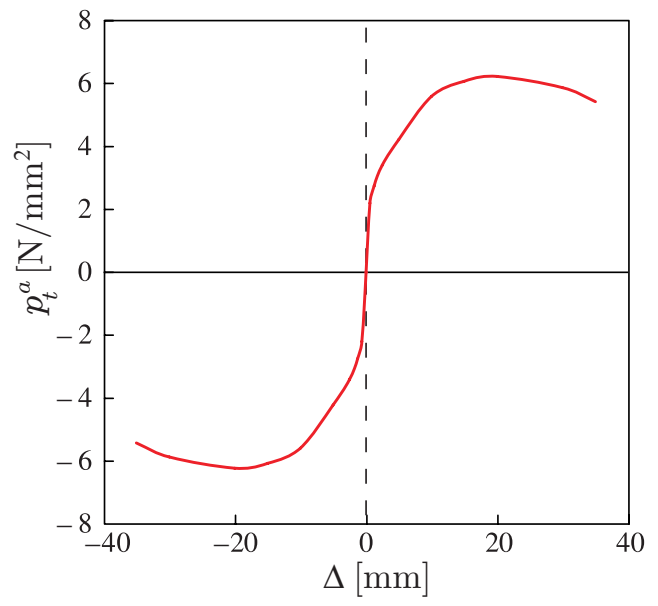

(b) normal traction-uplift relationship

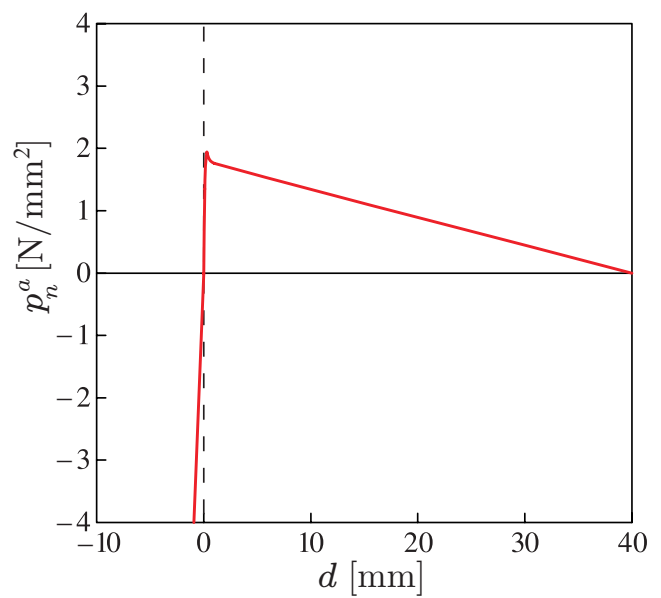

Fig. 7. Non-linear relationships between layers: (a) shear traction-slip $\left(p_{t}^{a}-\Delta\right)$ relationship, (b) normal traction-uplift $\left(p_{n}^{a}-d\right)$.

and I-beam specimens. Yet there is only a portion of the T-beam test results included in our comparisons.

The beams were constructed from spruce-pine-fir webs (38 x $89 \mathrm{~mm}$ x 244 mm) and different flanges (a 19 x $406 \mathrm{~mm}$ CDX plywood or a 11 x $406 \mathrm{~mm}$ oriented strandboard). 8d common nails spaced at $152 \mathrm{~mm}$ were used to fasten the flanges to the webs. A double layer of polyethylene was inserted in all slip planes to reduce the variability of friction at the contact between the layers. The beams were tested on a $2130 \mathrm{~mm}$ span and subjected to two point loadings $P_{2}=890$ N. Sufficiently low load levels were selected to ensure that the beam (web) stresses remained below their critical values.

Fig. 8 presents the loading arrangement on the beam and positions of slip $\left(A_{2}\right)$ and vertical deflection $\left(B_{2}\right)$ measuring spots.

Prior to the T-beam tests, material property tests (static bending) were run on the web specimens to determine web stiffness, sheatings (axial tension and compression to determine flange stiffnesses), and nails (lateral load/slip tests 


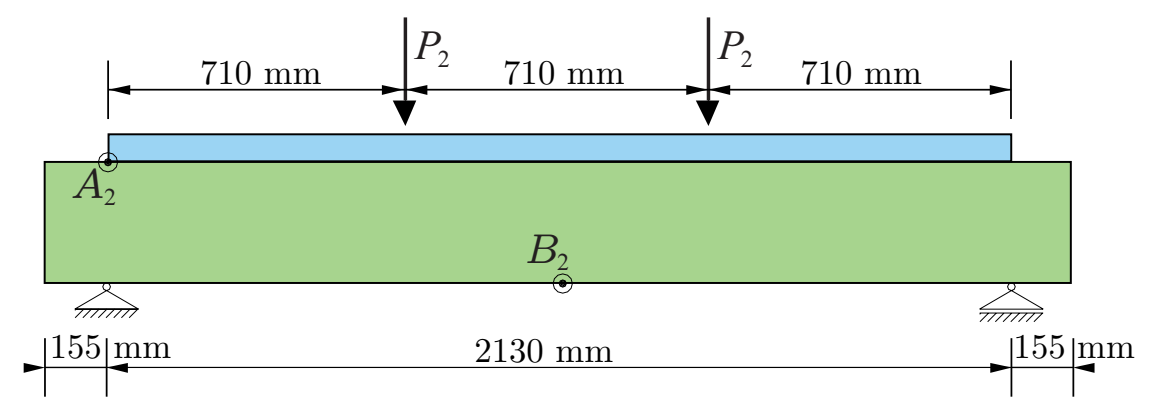

Fig. 8. Geometrical data, loading and position of slip $\left(A_{2}\right)$ and vertical deflection $\left(B_{2}\right)$ measuring.

with each type of sheating to determine interlayer slip properties). As a result of sheating test, an average elastic moduli of plywood $\left(E_{\text {flange }}=7708 \mathrm{~N} / \mathrm{mm}^{2}\right)$ and oriented standboard $\left(E_{\text {flange }}=3309 \mathrm{~N} / \mathrm{mm}^{2}\right)$ were estimated. The web elastic moduli considered in the numerical analysis are reported in the first column of Table 2 for each specimen. Fig. 9 presents the shear traction-slip curve used in the analysis. It was calculated from the typical load/slip curve for 8d nail, presented in McCutcheon [30]. There were no experimental data available to define the normal traction-uplift curve, however. We presume a rigid transverse connection between layers in our analysis.

\section{$5 \quad$ Numerical examples}

Several numerical examples are presented for verification and validation of the proposed approach. Verification of the mathematical model is performed: (1) by comparing numerical results to the analytical solution of linearly elastic beam [6], and (2) by comparing numerical solutions of a simply supported non-linear beam modelled by different finite element meshes.

Validation of the procedure is performed by comparing the numerical solution 


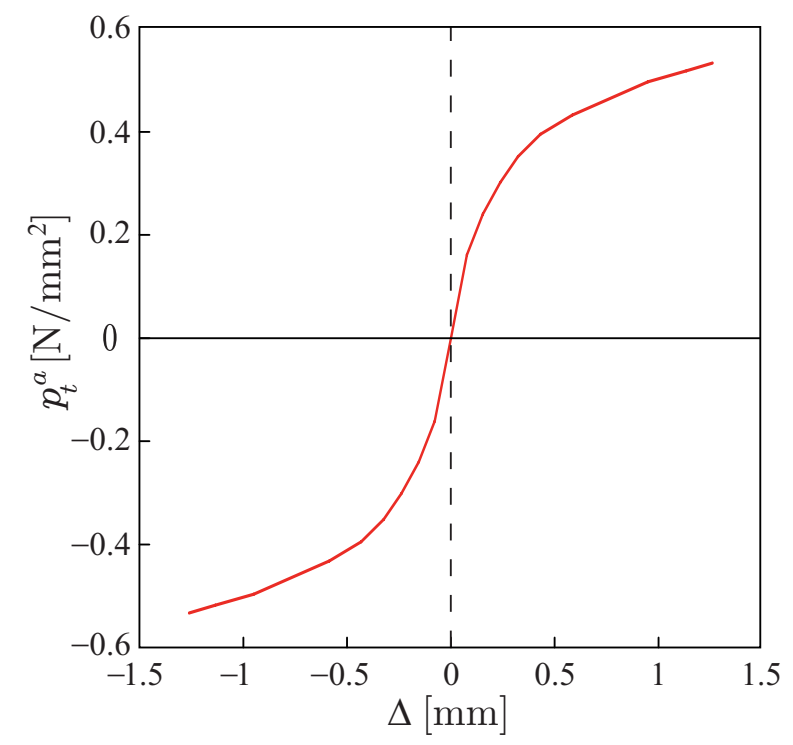

Fig. 9. Non-linear shear traction-slip relationship between layers (according to McCutcheon [30]).

first, with the experimental results of a full-scale laboratory test on a simply supported beam investigated by Planinc et al. [27] (Experiment A) and, second, with the experimental results of a full-scale laboratory test on a simply supported beam investigated by McCutcheon [30] (Experiment B). Presumed thickness of the connecting layer in all cases considered is $e=0 \mathrm{~mm}$. A further objective of Sec. 5 is to assess the effect of the transverse contact stiffness on mechanical behaviour of a two-layer timber beam.

\subsection{Verification of the mathematical model}

The suitability of the present numerical approach is verified by a simply supported beam with the same characteristics as previously described. In the first example, we consider a linear elastic behaviour of the beam. All characteristics of the beam were linearized around the undeformed configuration. 
We considered tangential slip modulus to be $K=352.5 \mathrm{~N} / \mathrm{mm}$. The constitutive law of the contact in the transverse direction was also linearized. After additionally assuming equal behaviour in tension and compression, we chose the tangential uplift modulus $C=1349.7 \mathrm{~N} / \mathrm{mm}$. The beam was loaded at the midspan with the point force $P=7624 \mathrm{~N}$ acting downwards. Each finite element has four interpolation points.

Fig. 10a presents relative errors of numerical solution of the midspan uplift with respect to the analytical solution (Kroflič et al. [6]) for different number of finite elements $N_{e}\left(\right.$ error $\left._{d, 1}=\frac{d_{A}^{\text {num }_{1}}-d_{A}^{\text {anal }}}{d_{A}^{\text {anal }}} 100 \%\right)$. It can be clearly seen that the model with only 6 finite elements gives accurate results.

(a) relative error of uplift $d_{A}$

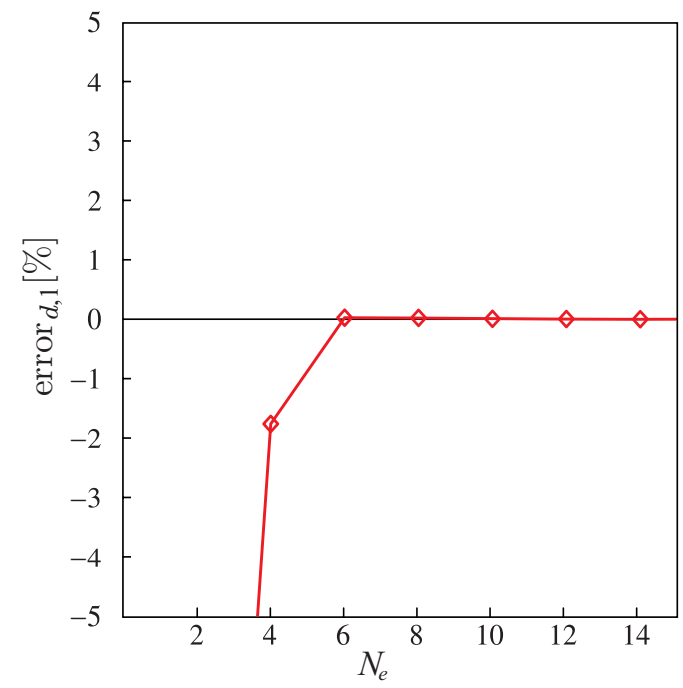

(b) relative error of $\operatorname{slip} \Delta_{B}$

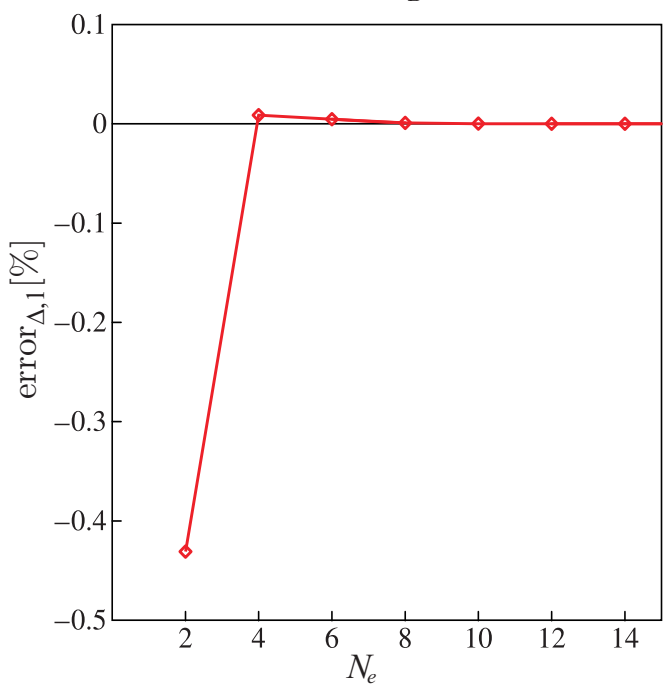

Fig. 10. Relative error of numerical solution (in per cent) at the contact of a simply supported elastic beam: (a) uplift at the midspan error $_{d, 1}$ and (b) slip error ${ }_{\Delta, 1}$ at the support.

Fig. 10b presents the comparison of relative errors of numerical solution at the edge of a simply supported beam with respect to the analytical solution of the slip $\left(\operatorname{error}_{\Delta, 1}=\frac{\Delta_{B}^{\text {num }_{1}}-\Delta_{B}^{\text {anal }}}{\Delta_{B}^{\text {anal }}} 100 \%\right)$. We can conclude that the model with 8 finite elements gives accurate results. 
The second example investigates the accuracy of the model when the loading approaches the ultimate loading of the beam and the behaviour becomes nonlinear. We considered experimentally obtained non-linear constitutive law of timber, shear traction-slip law and normal traction-uplift law at the contact. The value of ultimate force at the midspan of the beam found by the numerical analysis was $P=42800 \mathrm{~N}$. As the exact solution is not known, the results were compared to the solution obtained with 56 finite elements.

Fig. 11a presents relative errors of numerical solution of the midspan uplift with respect to the uplift obtained with the use of 56 finite elements $\left(\right.$ error $\left._{d, 2}=\frac{d_{A}^{\text {num }_{2}}-d_{A}^{56 \mathrm{FE}}}{d_{A}^{56 \mathrm{FE}}} 100 \%\right)$. Only a minor difference between the results obtained with 20 finite elements is found. Moreover, the difference in results is progressively smaller for a larger number of finite elements. Therefore the results for coarse finite element meshes suffice to be presented on the following figures.

(a) relative error of uplift $d_{A}$

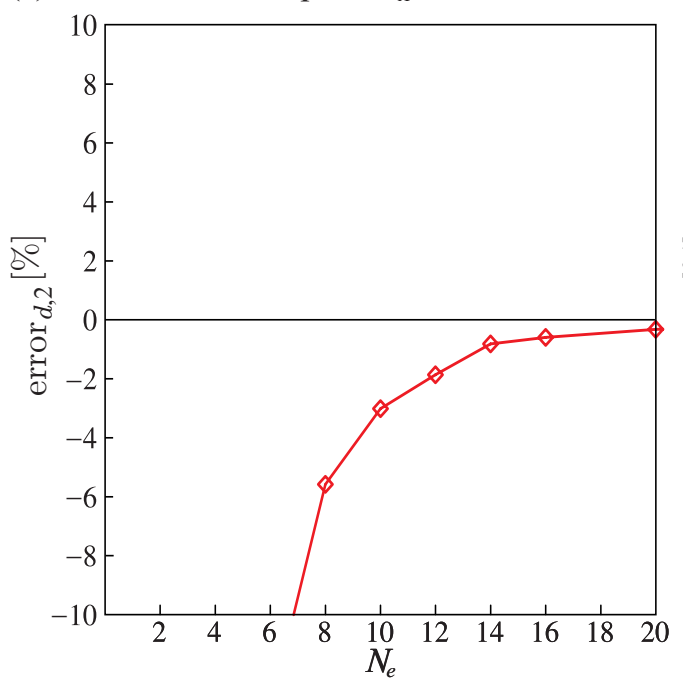

(b) relative error of slip $\Delta_{B}$

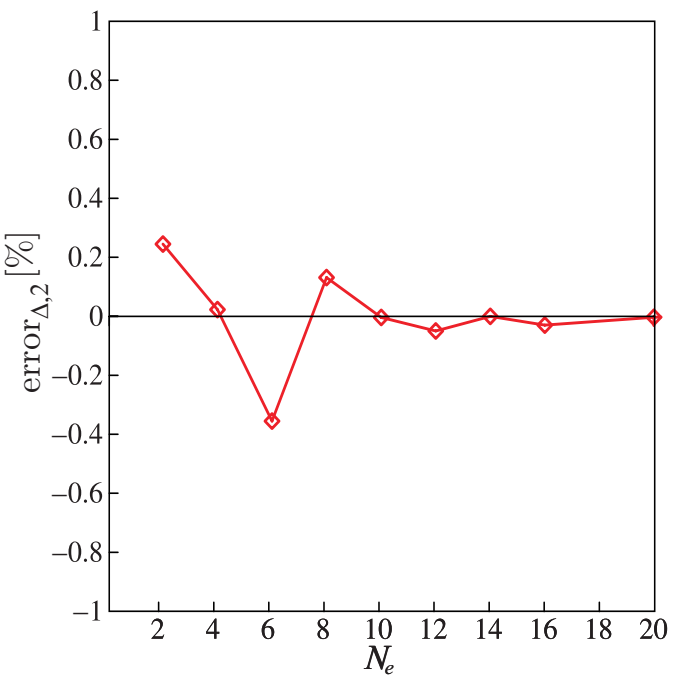

Fig. 11. Relative error of solution (in per cent) with respect to the results obtained with the use of 56 finite elements: (a) uplift at the midspan error $_{d, 2}$ and (b) slip $\operatorname{error}_{\Delta, 2}$ at the support. 
Fig. $11 \mathrm{~b}$ presents relative errors of numerical solution for slip $\Delta_{B}$ at the edge of a simply supported beam for different numbers of finite elements with respect to slip obtained with the use of 56 finite elements ( error $_{\Delta, 2}=$ $\left.\frac{\Delta_{B}^{\text {num }_{2}}-\Delta_{B}^{56 \mathrm{FE}}}{\Delta_{B}^{56 \mathrm{FE}}} 100\right)$. It can clearly be seen that there is only a minor deviation between the two results. Therefore we only present results for the 20 finite element mesh.

To clearly verify and strengthen numerical aspects of the present numerical model, we further study the linear elastic example studied previously, yet with the $\mathrm{T}$ cross-section (Fig. 12).

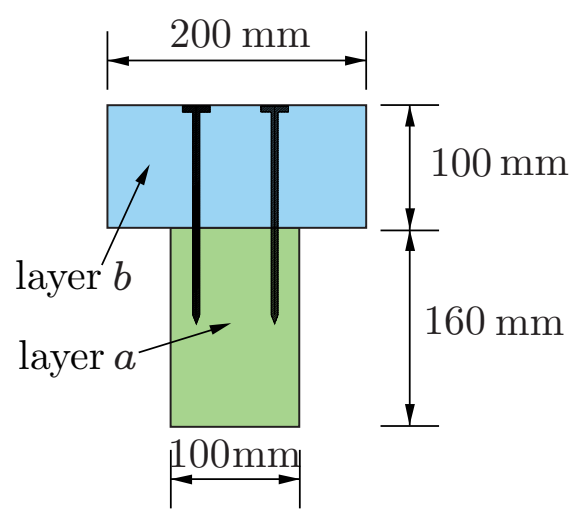

Fig. 12. T cross-section.

All the remaining geometric and material characteristics of the beam stays the same.

We consider several combinations of the tangential slip modulus, $K$, and the tangential uplift modulus, $C$ :

- $K=0, C=1349.7 \mathrm{~N} / \mathrm{mm}$ (without shear connection - WSC)

- $K \approx \infty, C=1349.7 \mathrm{~N} / \mathrm{mm}$ (rigid shear connection - RSC)

- $K \approx \infty, C \approx \infty$ (completely rigid connection - CRC). 
It is worth mentioning that, for the case without shear connection, we had to additionally support the upper beam layer to be able to satisfy boundary conditions of a two-layer beam. The numerical solutions are compared with the analytical solutions (Kroflič et al. [6]) for a different number of finite elements $N_{e}$.

The results for the vertical deflection $w_{A}$ of the midpoint of the bottom layer are presented in Table 1 . As a result of a good convergence, we present only relative errors for $4\left(\right.$ error $\left._{4 F E}\right)$ and $8\left(\right.$ error $\left._{8 F E}\right)$ element meshes.

Table 1

Analytical and numerical results and convergence properties of the midpoint vertical deflection for different shear and uplift connections.

\begin{tabular}{ccccc} 
Cross-section & Connection & Analytical $[\mathrm{mm}]$ & error $_{4 F E}[\%]$ & $\operatorname{error}_{8 F E}[\%]$ \\
\hline Original & WSC & 10.5682 & -0.08020 & -0.08155 \\
Original & RSC & 4.43766 & -0.67189 & -0.67119 \\
Original & CRC & 4.42244 & -0.00289 & -0.00327 \\
\hline $\mathrm{T}$ & WSC & 5.98058 & -0.62352 & -0.62336 \\
$\mathrm{~T}$ & $\mathrm{RSC}$ & 1.55624 & -2.66826 & -2.66452 \\
$\mathrm{~T}$ & $\mathrm{CRC}$ & 1.51032 & -0.01377 & -0.01893 \\
\hline
\end{tabular}

Considered tolerance of Newton method is $10^{-8}$ in all presented cases. All numerical results are within expected limits. There is a small convergence problem noticed in the sense of decrease of an error with growing number of finite elements. Especially, in the case of rigid shear connection (RSC) relative errors stabilize and do not decrease with growing number of finite elements. 


\subsection{Validation of the mathematical model}

- Experiment A

Deflections and slip between layers were measured at several points along the beam axis.

Fig. 13a compares the measured and the calculated load-deflection curves at the midspan for the bottom layer $a$. All experimentally obtained characteristics of the tested beam were considered in the numerical analysis: the non-linear material model for timber, the non-linear shear traction-slip and the non-linear normal traction-uplift relationships.

An excellent agreement between measured and calculated load-displacement curves can be observed at all load levels. The experimentally observed collapse mechanism of the beam agrees with the collapse mechanism found in our numerical calculation which showed that the collapse occurred due to tensile failure of timber fibres at the bottom of layer $a$ at the midspan.

The experimentally obtained ultimate loading is $P_{\mathrm{ult}}^{\exp }=43600 \mathrm{~N}$, whereas the numerically obtained one is $P_{\text {ult }}^{\text {num }}=42900 \mathrm{~N}$. The finite elements that were employed are denoted by $E_{j-k}$, where the first subscript $(j)$ indicates the number of interpolation points and $(k)$ the number of the Gaussian integration nodes along the beam length. The numerical results shown here were obtained with the mesh having 20 finite elements per one half of the beam.

Fig. 13b presents the comparison between the measured and the calculated load--end slip curves. The end slip was measured just at the end points of the beam. These points were marked as IND1 (left end) and IND2 (right end). A good general agreement could be observed again, although the measured load-end slip curves differ somewhat this time. Note that the scatter 
(a) load-deflection curve

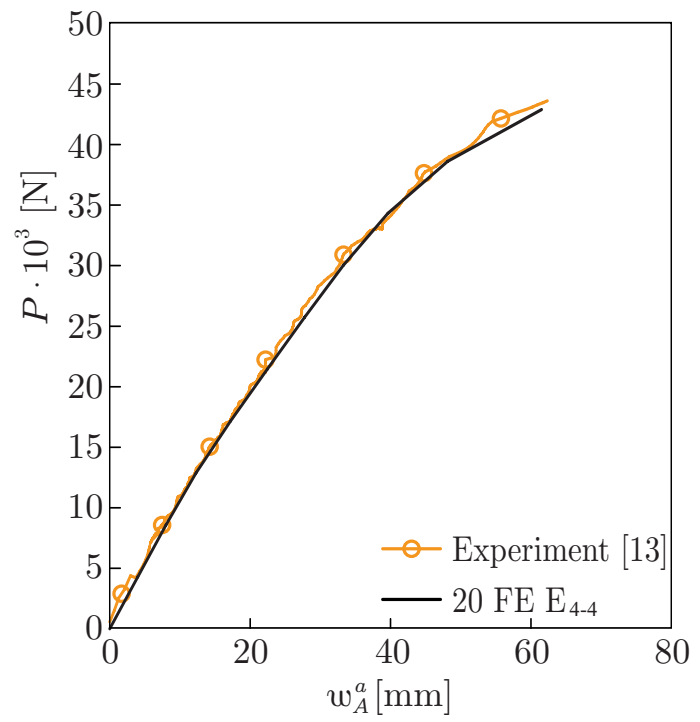

(b) load-slip curve

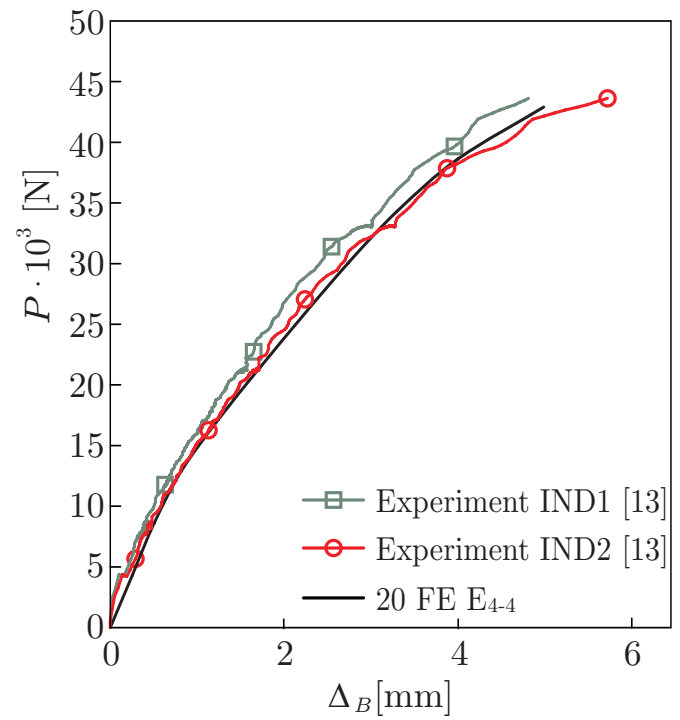

Fig. 13. Measured and calculated (a) load-deflection $\left(P-w_{A}^{a}\right)$ and (b) load-slip $\left(P-\Delta_{B}\right)$ curves.

of the experimental results could be the reason for these small discrepancies.

The measured (full line) and the calculated (circles) deformed shapes are presented in Fig. 14. A very good agreement between the measured and the calculated deformed shapes can be observed for all load levels. 


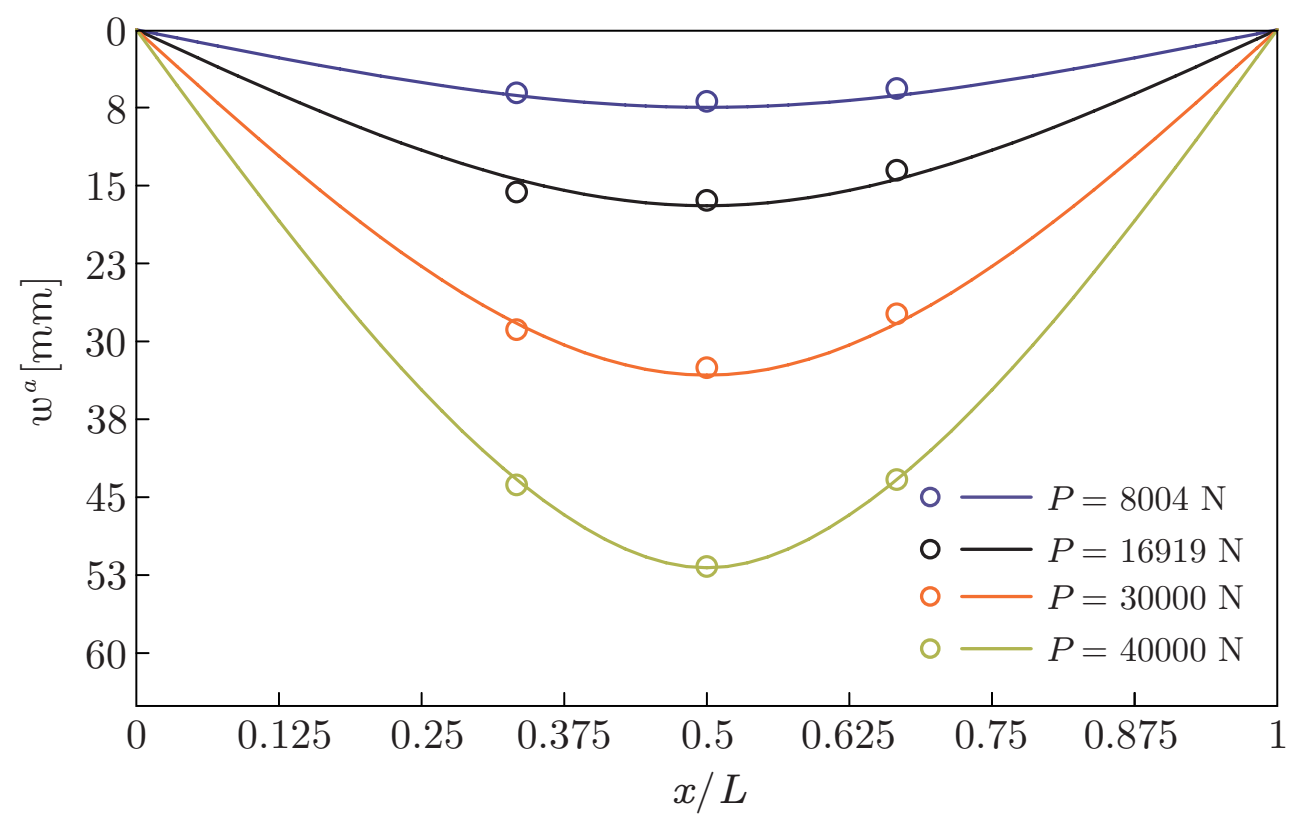

Fig. 14. Measured and calculated deformed shapes of the beam at different load levels.

- Experiment B

In the original experiment, three different specimens were tested for each type of the flange. Comparisons of the experimental and numerical results are presented in Table 2.

The above comparisons indicate a good agreement among the experimental and numerical results. Some deviations may be the result of averaged values of elastic modulus of the flange used in numerical calculations.

\subsection{The effect of transverse stiffness of connecting layer}

It is interesting to study the effect of transverse stiffness of the connecting layer on the static and kinematic quantities in the two-layer timber beam. The influence of the normal traction-uplift constitutive law on the beam analysed 
Table 2

Comparisons of analytical and numerical results; results in $\left[\mathrm{N} / \mathrm{mm}^{2}\right]$ (elastic modulus) and [mm] (vertical deflection, slip).

\begin{tabular}{cccccccc}
\hline \hline Flange & $E_{w e b}$ & $w_{B_{2}}^{\text {test }}$ & $w_{B_{2}}^{\text {num }}$ & $\operatorname{error}_{w}[\%]$ & $\Delta_{A_{2}}^{\text {test }}$ & $\Delta_{A_{2}}^{\text {num }}$ & $\operatorname{error}_{\Delta}[\%]$ \\
\hline PLY & 10342 & 8.961 & 8.865 & 1.07 & 0.603 & 0.6062 & -0.53 \\
& & & & & & & \\
PLY & 9446 & 9.745 & 9.54 & 2.10 & 0.6891 & 0.652 & 5.38 \\
& & & & & & & \\
PLY & 8687 & 10.37 & 10.22 & 1.45 & 0.739 & 0.698 & 5.55 \\
\hline OSB & 9791 & 10.599 & 9.68 & 8.67 & 0.64 & 0.623 & 2.66 \\
OSB & 11307 & 9.65 & 8.57 & 11.19 & 0.574 & 0.549 & 4.36 \\
OSB & 11170 & 8.84 & 8.66 & 2.04 & 0.509 & 0.555 & -9.04 \\
\hline
\end{tabular}

in Sec. 5.2 was studied first. Then, the same procedure is applied on a continuous, statically indetermined two-span beam. In all cases we consider the realistic non-linear shear traction-slip $\left(p_{t}^{a}-\Delta\right)$ relationship for arrangement of nails at distance $230 \mathrm{~mm}(\mathrm{~N} 23)$.

There are three types of the nail arrangement considered in vertical direction: nails applied at distances $40 \mathrm{~mm}$, resulting in a rather stiff contact (N4), $230 \mathrm{~mm}$ (N23) and $460 \mathrm{~mm}$, a very flexible contact (N46). Furthermore, in order to assess the compressive stiffness of the connection, we reduce the compression part of the normal traction-uplift constitutive law N46 by factors 0.1 $\left(10 \%, \mathrm{~N} 46_{\mathrm{red}_{10 \%}}\right)$ and $0.01\left(1 \%, \mathrm{~N} 46_{\mathrm{red}_{1 \%}}\right)$ of the original (i.e. experimentally obtained) value (Fig. 15). Considering such a variety of constitutive relationships in transverse direction, results in an extensive review of influence on the rest of the quantities of a composite beam. Most of them correspond to feasible 
values for composite timber beams. Although some of the considered values of the transverse stiffness may have a questionable physical significance, the results prove a wide range of applicability of the method.

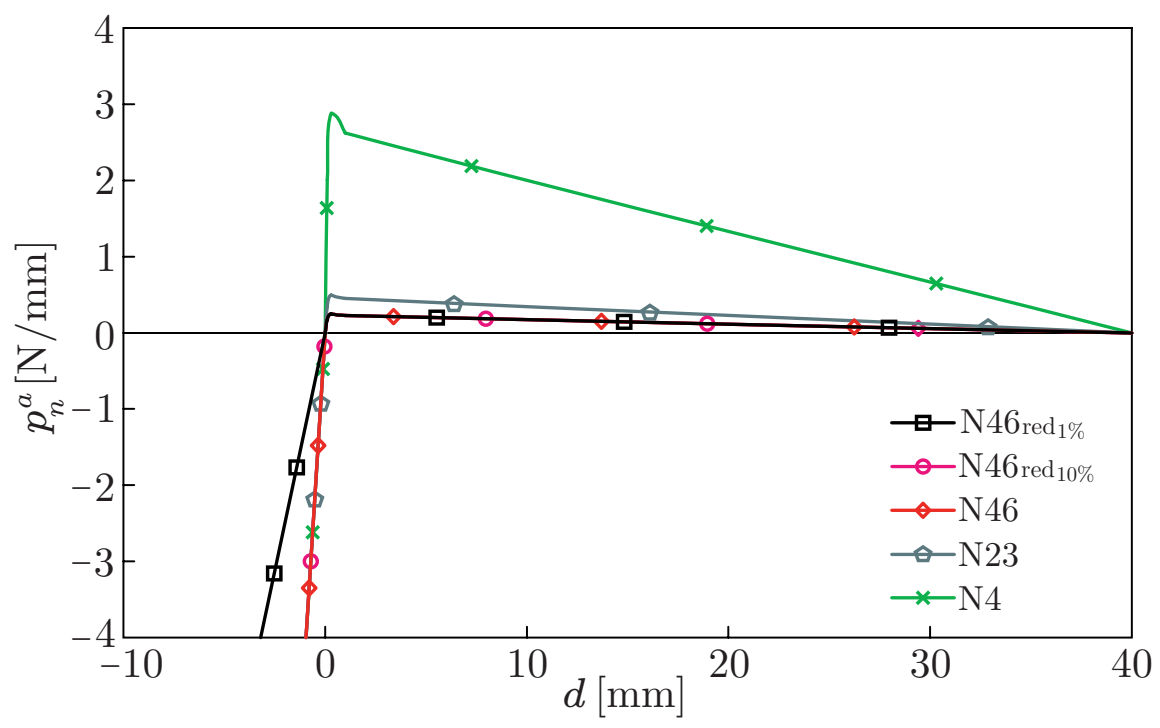

Fig. 15. Non-linear normal traction-uplift constitutive laws in the parametric study.

\subsubsection{Simply supported beam}

In order to assess the effect of the point of application of the load, we consider two cases of point load $P=34900 \mathrm{~N}$ acting at the midspan of the beam considered in Sec. 5.2: $(i)$ the load is acting on the upper layer, and $(i i)$ the load is acting on the bottom layer.

- Point load at the midspan of the upper layer

First we examine the influence of the variable normal traction-uplift constitutive law on slip $(\Delta)$ and uplift $(d)$. Fig. 16a presents the influence of the non-linear normal traction-uplift constitutive law on slip $\Delta$. It is clearly seen that the effect is only minor. 
Fig. 16b presents the influence of the non-linear normal traction-uplift constitutive law on uplift $d$. We notice that the reduction of the normal compression stiffness has a significant influence on uplift $d$; in contrast, the basic nail arrangements behave roughly the same.

(a) $\operatorname{slip} \Delta$ at the contact of layers

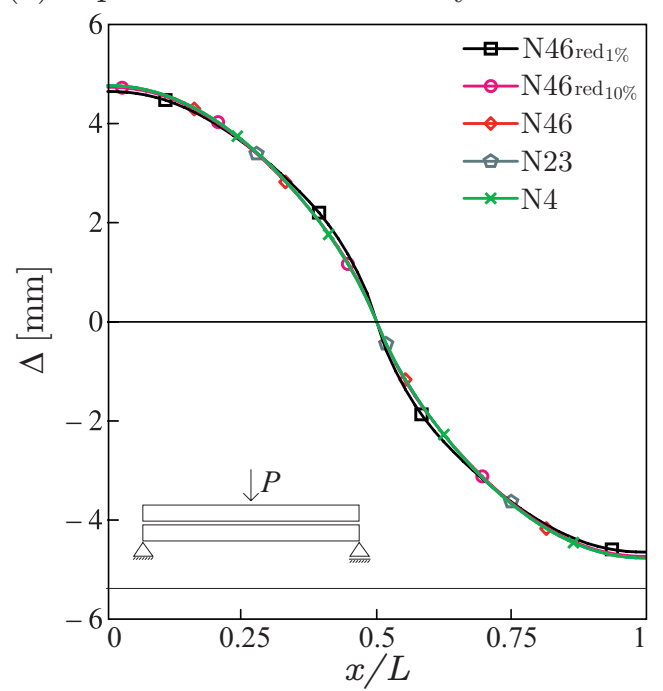

(b) uplift $d$ at the contact of layers

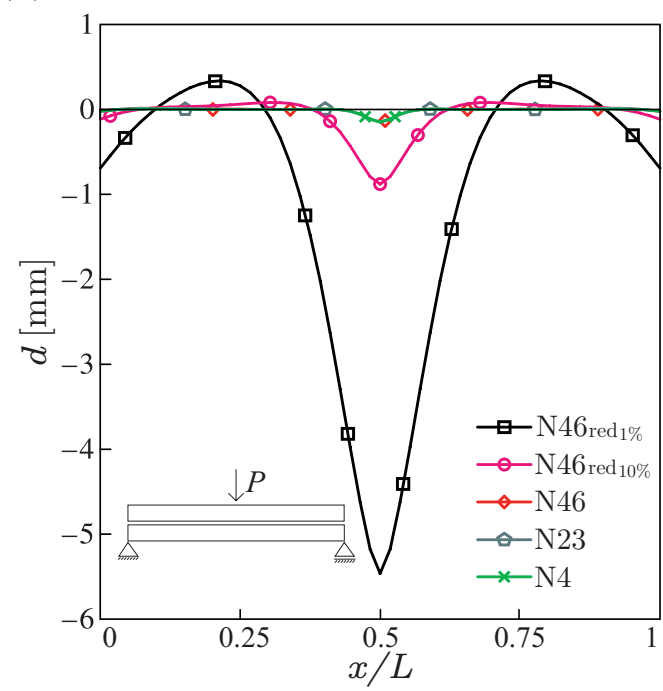

Fig. 16. Slip $\Delta$ and uplift $d$ distributions along the contact surface for different nail arrangements and different types of the normal contact traction-uplift relationships (load acting on the upper layer).

Fig. 17a presents the influence of the non-linear normal traction-uplift constitutive law on shear force $Q^{a}$ in the bottom layer. As for uplift $d$, there is no effect of the considered arrangements of the nails. By contrast, the reduced normal contact laws result in a significant change of the bottom layer shear force $Q^{a}$ distribution in the vicinity of the point of application of the load. Fig. 17b presents the influence of the non-linear normal traction-uplift constitutive law on the upper layer shear force $Q^{b}$. Again the influence on $Q^{b}$ is substantial, yet only in the region of the point of application of the load and if a reduced normal traction-uplift constitutive law is used. 
(a) shear force $Q^{a}$ in lower layer

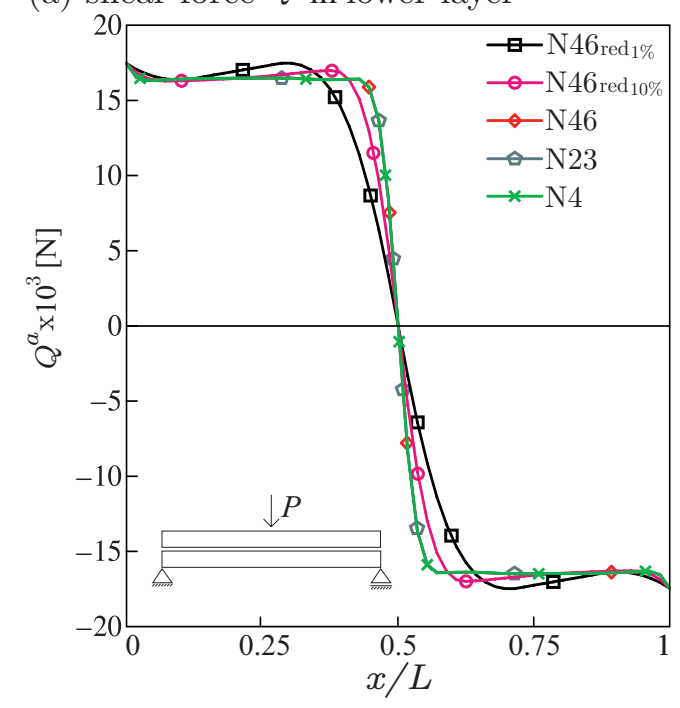

(a) shear force $Q^{b}$ in upper layer

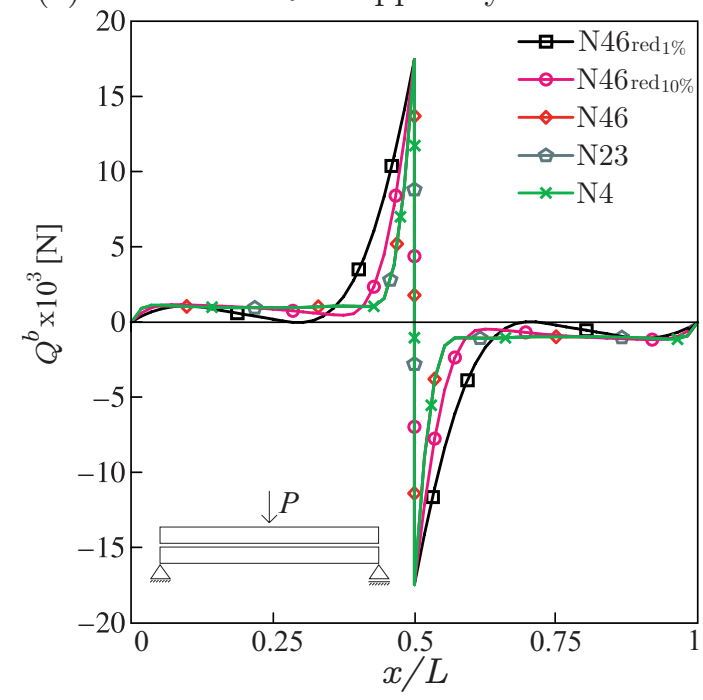

Fig. 17. Influence of different normal contact traction-uplift relationships on the shear forces $Q^{a}$ and $Q^{b}$ (load acting on the upper layer).

- Point load at the midspan of the bottom layer

Fig. 18a shows that, again, the influence of the non-linear normal tractionuplift constitutive law on slip $\Delta$ is minor.

Fig. 18b shows the effect of the non-linear normal traction-uplift law on uplift d. This time a considerable influence of the basic nail arragements on the uplift is observed. The further reduction of normal traction-uplift law in compression has a negligible effect.

Fig. 19a presents the effect of the non-linear normal traction-uplift law on the bottom layer shear force $Q^{a}$. We may observe that the effect of different contact relations is small. Fig. 19b depicts these effects on the upper layer shear force $Q^{b}$. Different arrangements of nails have a significant effect on the distribution of the upper layer shear force $Q^{b}$. Similar results have also been obtained for bending moments in the bottom and upper layers. 
(a) $\operatorname{sip} \Delta$

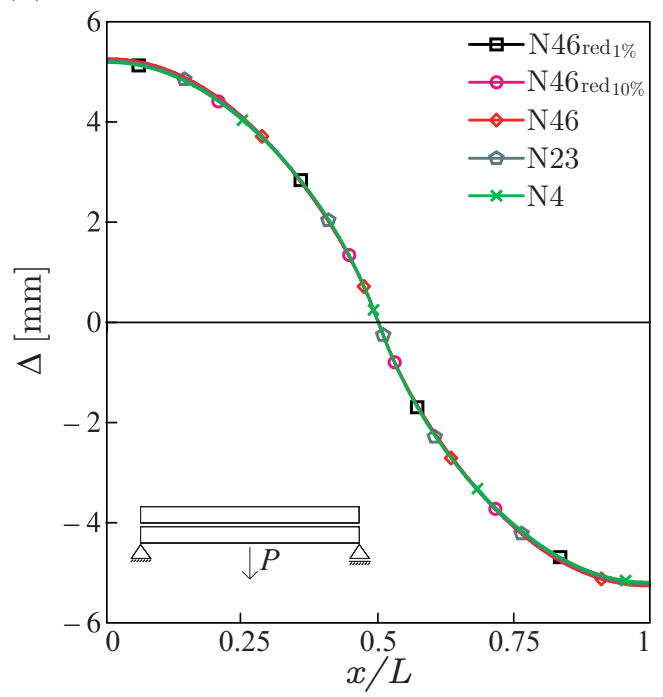

(b) uplift $d$

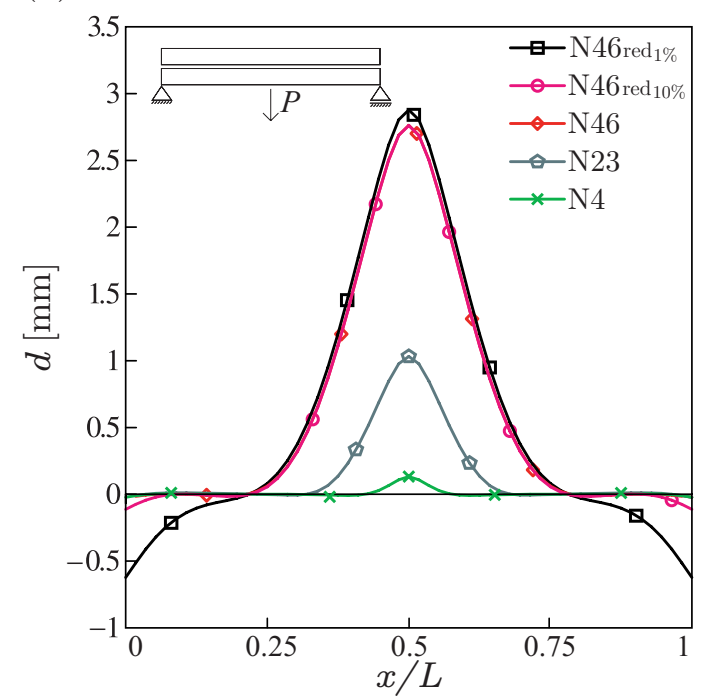

Fig. 18. (a) Slip $(\Delta)$ and (b) uplift $(d)$ distribution along the contact for different types of normal contact traction-uplift relationships (load acts on the bottom layer).

(a) shear force $Q^{a}$ in lower layer

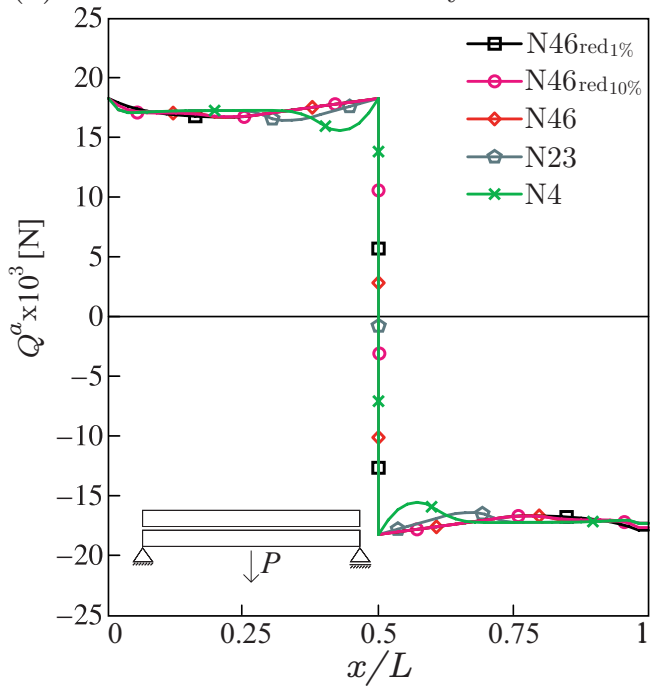

(a) shear force $Q^{b}$ in upper layer

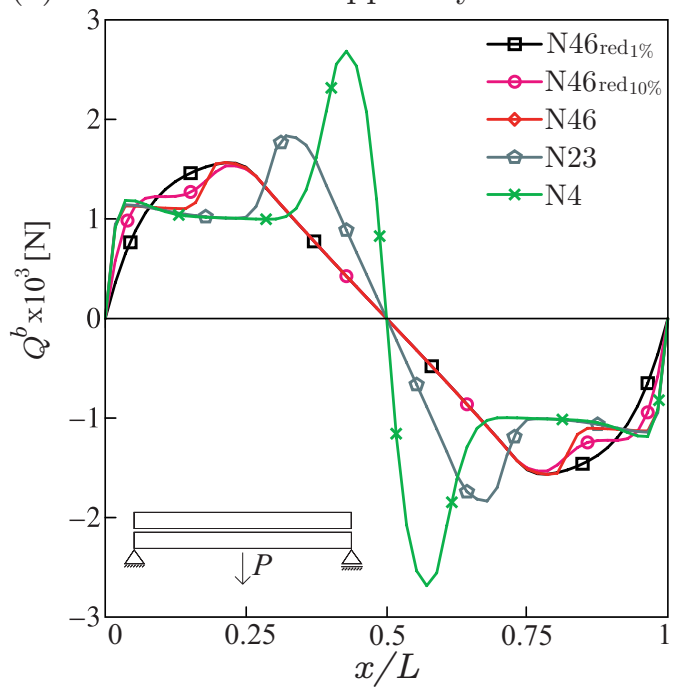

Fig. 19. Influence of different normal contact traction-uplift relationships on shear forces $Q^{a}$ and $Q^{b}$ (load acts on the bottom layer).

\subsubsection{Continuous two-span beam}

We consider two cases of the point load $P=33700 \mathrm{~N}$ acting at the midpoint of the first span of a continuous beam: $(i)$ the load acts at the upper layer 
downwards and $(i i)$ the load acts at the bottom layer downwards. The total length of the continuous beam is $L=7000 \mathrm{~mm}$. The lengths of the particular spans are $L_{1}=4000 \mathrm{~mm}$ and $L_{2}=3000 \mathrm{~mm}$. All the remaining material and geometrical characteristics are those of the simply supported beam, presented in Sec. 5.2.

- Point load at the midspan of the upper layer

Fig. 20a shows the influence of the normal contact traction laws on slip $\Delta$. It is clearly seen that the effect is negligible. Fig. 20b presents effects on uplift $d$. As observed from the figure, the results of all three basic nail arrangements practically coincide. In contrast, the reduction of the normal contact law in compression largely affects uplift $d$, particularly in the vicinity of the point of application of the load.

(a) $\operatorname{slip} \Delta$

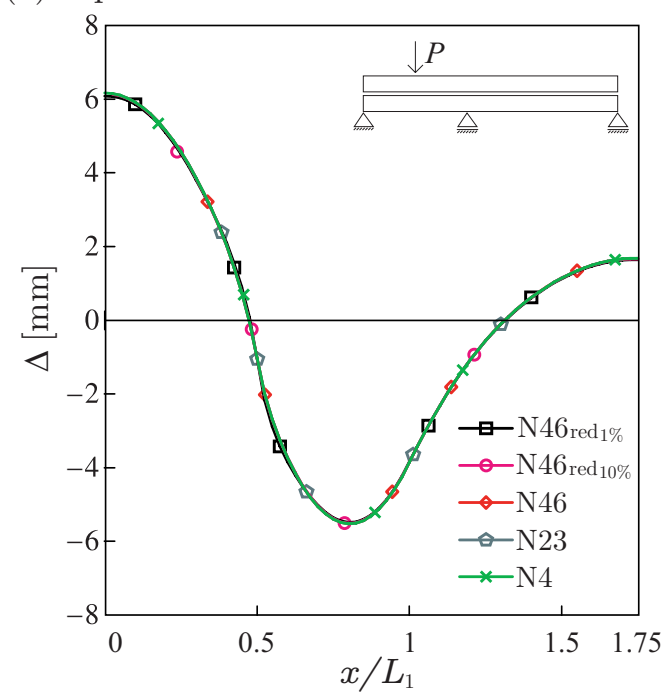

(b) uplift $d$

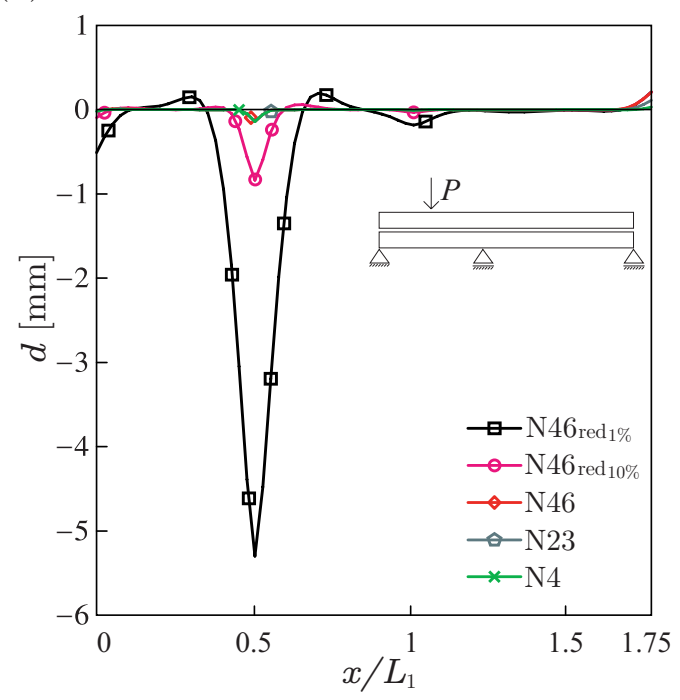

Fig. 20. (a) Slip $(\Delta)$ and uplift $(d)$ distribution along the contact for different types of normal contact traction-uplift relationships (force acts on the upper layer).

Fig. 21a shows that the influence of the three basic nail arrangements on the bottom layer shear force $Q^{a}$ is negligible. The reduced normal contact laws 
results in a small change of the bottom layer shear force $Q^{a}$ distribution, yet only locally. Fig. 21b displays effects on the upper layer shear force $Q^{b}$. Again, the effect on $Q^{b}$ is localized to the vicinity of the point of application of the load and is considerable only for reduced normal contact laws.
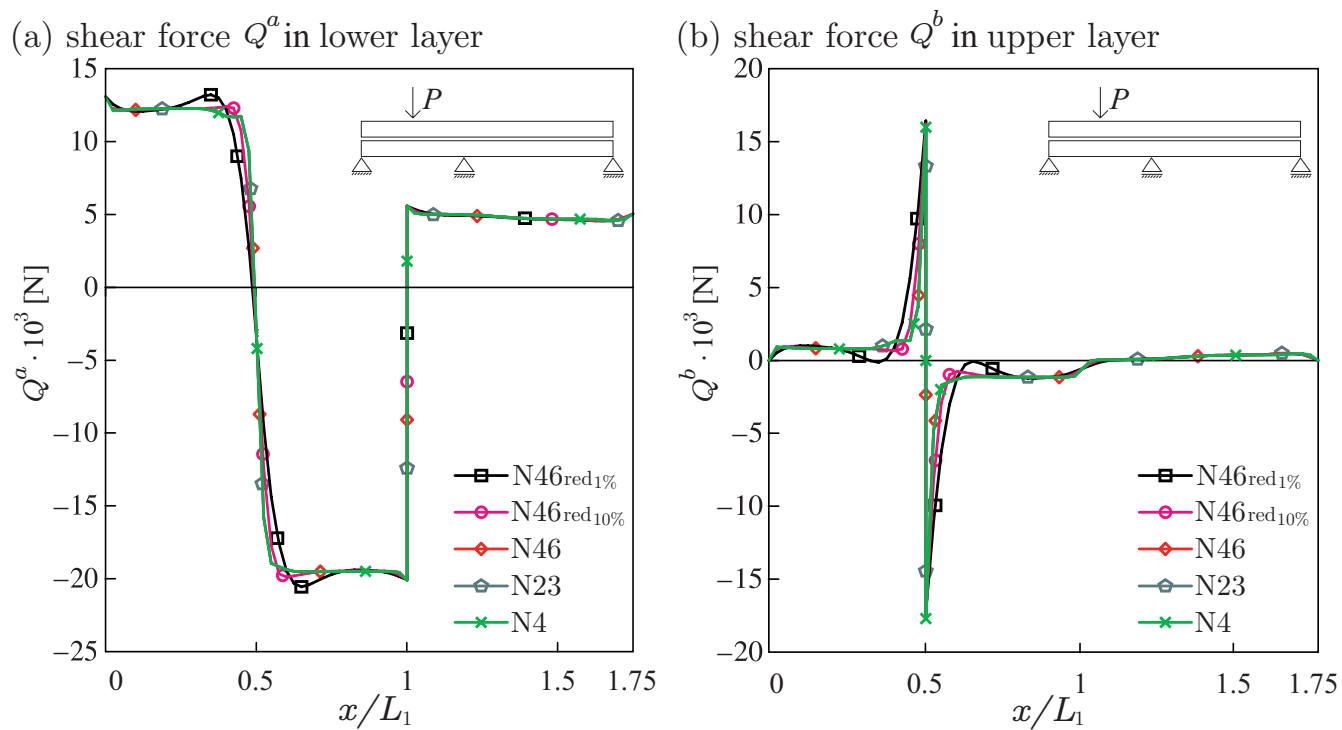

Fig. 21. Influence of different normal contact traction-uplift relationships on shear forces $Q^{a}$ and $Q^{b}$ (force acts on the upper layer).

- Point load at the midspan of the bottom layer

Fig. 22a shows that the influence of the non-linear normal traction-uplift constitutive law on slip $\Delta$ in a continuous beam is negligible.

Fig. 22b shows that the influence on uplift $d$ is considerable, particularly with regard to the arrangements of nails.

Fig. 23 presents results for shear force $Q$. Fig. 23a displays the variation of the bottom layer shear force $Q^{a}$, and Fig. 23b for the upper layer shear force $Q^{b}$. As observed, different arrangements of nails can have a substantial influence on the distribution of the upper layer shear force $Q^{b}$. Similar results have also 
(a) $\operatorname{slip} \Delta$

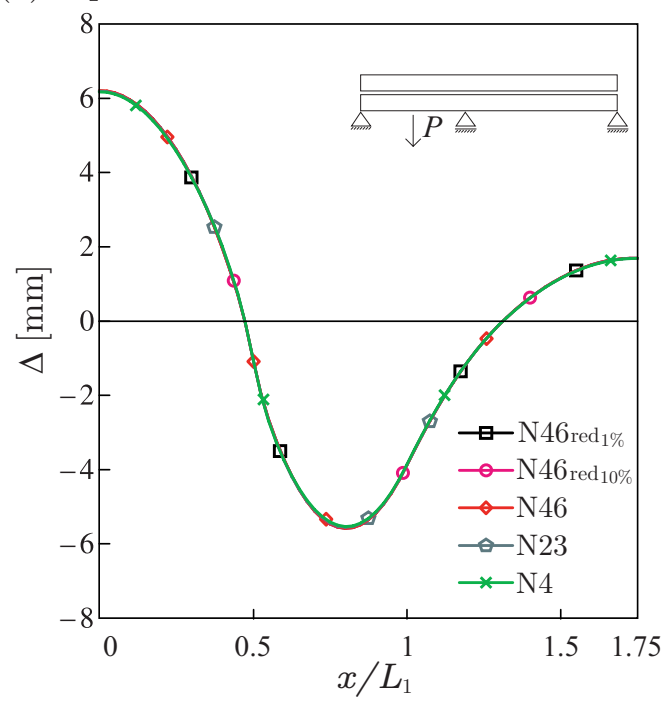

(b) uplift $d$

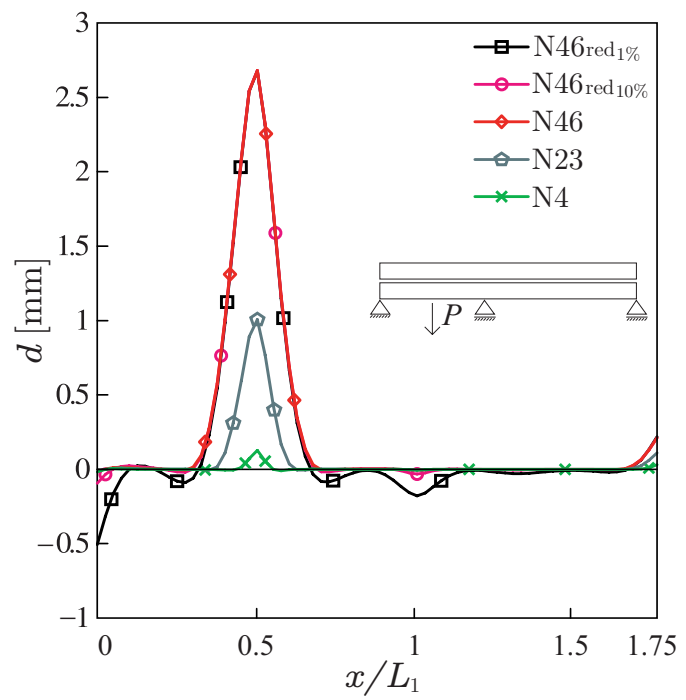

Fig. 22. Slip $\Delta$ (uplift $d$ ) distribution along the contact for different types of normal contact traction-uplift relationships (force acts on the upper layer).

been obtained regarding the distribution of bending moments in the layers.

(a) shear force $Q^{a}$ in lower layer

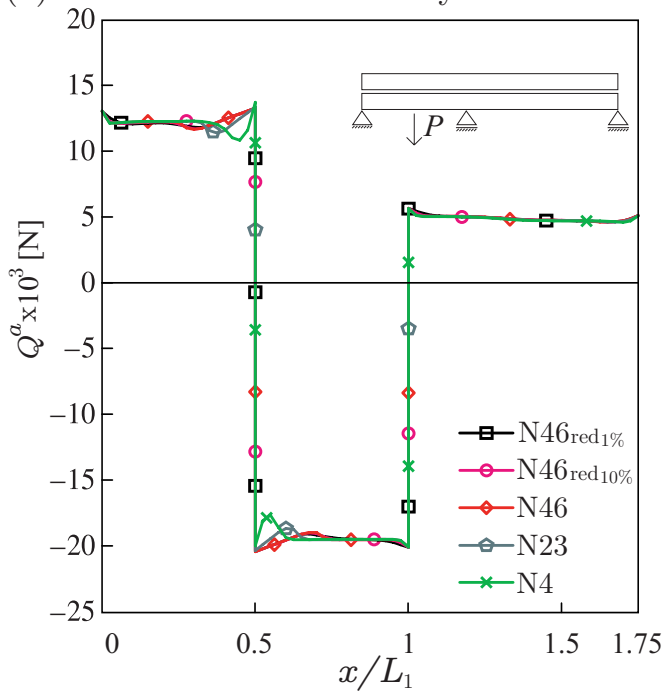

(b) shear force $Q^{b}$ in upper layer

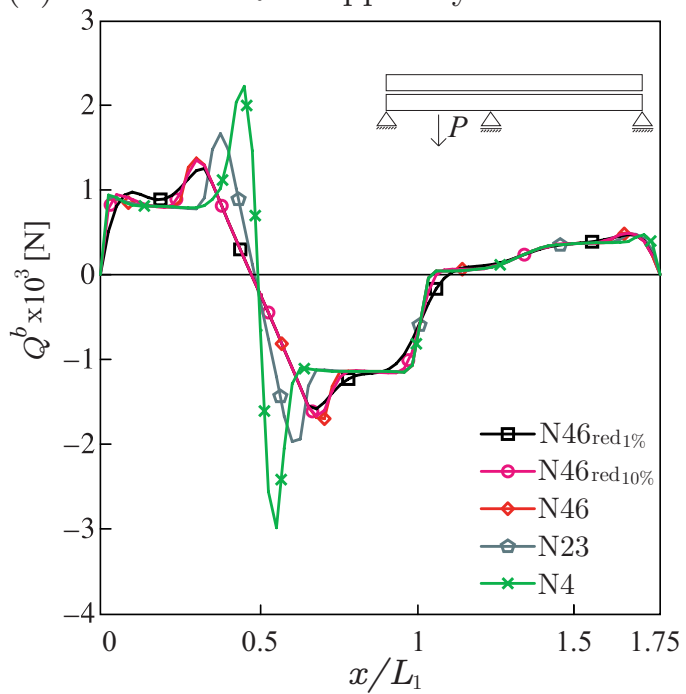

Fig. 23. Influence of different normal contact traction-uplift relationships on the shear forces $Q^{a}$ and $Q^{b}$ (force acts on the upper layer).

We have seen that the effect of the normal contact traction law on behaviour of beams is not negligible where considerable variations in results can be ob- 
served.

\section{Conclusion}

A new mathematical model and its numerical solution method for the analysis of two-layer timber beams was presented. We derived an effective, strain-based finite element method for the non-linear analysis of two-layer timber beams with flexible connections experiencing interlayer slip and uplift. The formulation considers the geometrically linear and materially non-linear planar beam theory. The shear traction-slip and the normal traction-uplift relationships of the contact surface as well as the stress-strain relationship of the timber are taken to be non-linear.

The suitability of the present numerical approach was verified by two numerical examples, considering linear and non-linear material behaviour. The convergence of the proposed numerical method was found appropriate. Validity, accuracy and reliability of the model was studied by comparing the numerical results with the experimentally obtained ones of McCutcheon [30] and Planinc et al. [27]. An excellent agreement between the measured and the calculated results is observed for all load levels. After the verification and the validation have been concluded, it was clear that the present new mathematical model and its solution method represent a suitable practical tool for the analysis of two-layer timber beams with a partial interface connection.

The influence of different normal traction-uplift constitutive relationships on the kinematic and static quantities of simply supported and continuous beams was investigated in detail. It was observed that, for the range of the realistic 
normal traction-uplift constitutive relationships in timber structures employed in the analysis, the connection in normal direction was relatively rigid. In contrast, the flexible contact relationship can have a visible influence on the uplift. In some cases, there was also a considerable effect detected on the distribution of shear forces and bending moments along the composite beam.

A significant differences in the uplift distribution may appear between the cases, when the point of application of the force was on the top or on the lower layer. If the top layer is loaded, uplift is negative and highly localized in the loaded zone. When the load acts on the bottom layer, uplift is still highly localized in the loaded zone, but is positive.

It can be concluded that the effect of the normal contact traction-uplift constitutive relationship on most of the kinematic and static quantities is negligible. In some rare cases, however, appreciable variations in results for uplift, and internal and contact forces can be observed.

\section{Acknowledgment}

This work was supported by the Slovenian Research Agency through the grant 1000-07-310191. The support is gratefully acknowledged.

\section{References}

[1] H. Granholm, On composite beams and columns with special regard to nailed timber structures, Trans. No. 88, Chalmers University of Technology, Goeteborg, Sweden (In Swedish), 1949. 
[2] P. F. Pleshkov, Theoretical studies of composite wood structures, Soviet Union (In Russian), 1952.

[3] F. Stüssi, Zusammengesetze Vollwandträger, International Association for Bridge and Structural Engineerig (IABSE) 8, 249-269, 1947 (in German).

[4] N. M. Newmark, C. P. Siess, I. M. Viest, Tests and analysis of composite beam with incomplete interaction, Proc. Society for Experimental Stress Analysis 9, 75-92, 1951.

[5] U. A. Girhammar, V. K. A. Gopu, Composite beam-columns with interlayer slip - exact analysis, ASCE Journal of Structural Engineering 119(4), 1265-1282, 1993.

[6] A. Kroflič, I. Planinc, M. Saje, B. Čas, Analytical test solution of two-layer beam including interlayer slip and uplift, University of Ljubljana, Faculty of Civil and Geodetic Engineering, Internal Report, 2008.

[7] G. Ranzi, M. A. Bradford, B. Uy, A general method of analysis of composite beam with partial interaction, Steel and Composite Structures 3(3), 169-184, 2003.

[8] S. Schnabl, I. Planinc, M. Saje, B. Čas, G. Turk, An analytical model of layered continuous beams with partial interaction, Structural Engineering and Mechanics 22(3), 263-278, 2006.

[9] S. Schnabl, M. Saje, G. Turk, I. Planinc, Locking-free two-layer Timoshenko beam element with interlayer slip, Finite Elements in Analysis and Design 43(9), 705-714, 2007.

[10] A. Ayoub, A force-based model for composite steel-concrete beams with partial interaction, Journal of Constructional Steel Research 61(3), 387-414, 2005.

[11] R. Betti, A. Gjelsvik, Elastic composite beams, Computers and Structures 59(3), 437-451, 1996. 
[12] B. Čas, M. Saje, I. Planinc, Non-linear analysis of composite steel-concrete beams with incomplete interaction, Steel and Composite Structures 4(6), 489$507,2004$.

[13] N. Gattesco, Analytical modeling of nonlinear behavior of composte beams with deformable connection, Journal of Constructional Steel Research 52, 195-218, 1999.

[14] M. J. S. Hirst, M. Y. Yeo, The analysis of composite beams using standard finite element programs, Computers and Structures 11(3), 233-237, 1980.

[15] G. Ranzi, M. A. Bradford, Direct stiffness analysis of a composite beam-column element with partial interaction, Computers and Structures 85(15-16), 1206$1214,2007$.

[16] H. Y. Rassam, Y. R. Goodman, Buckling behavior of layered wood columns, Wood Science 2(4), 238-246, 2007.

[17] R. Salari, E. Spacone, P. B. Shing, D. M. Frangopol, Nonlinear analysis of composite beams with deformable shear connectors, ASCE Journal of Structural Engineering 124(10), 1148-1158, 1998.

[18] R. Seracino, D. J. Oehlers, M. F. Yeo, Partial-interaction flexural stresses in composite steel and concrete bridge beams, Engineering Structures 23, 11861193, 2001.

[19] E. G. Thompson, J. R. Googman, M. D. Vanderbilt, Finite element analysis of layered wood system, Journal of Structural Division, Proceeding ASCE 101(ST12), 2659-2672, 1975.

[20] B. Čas, M. Saje, I. Planinc, Non-linear finite element analysis of composite planar frames with an interlayer slip, Computers and Structures 82, 1901-1912, 2004 . 
[21] T. Hozjan, Nonlinear analysis of composite planar structures exposed to fire, University of Ljubljana, Faculty of civil and geodetic engineering, $\mathrm{Ph}$. D. thesis, (in Slovene), 2009.

[22] A. O. Adekola, Partial interaction between elastically connected elements of a composite beam, International Journal of Solids and Structures 4(11), 11251135, 1968.

[23] H. Robinson, K. S. Naraine, Slip and uplift effects in composite beams, International Conference on Composite Construction in Steel and Concrete. Proceedings of an Engineering Foundation Conference ASCE, New England College, Henniker, New Hampshire, 487-497, 1988.

[24] F. Gara, G. Ranzi, G. Leoni, Displacement based formulations for composite beams with longitudinal slip and vertical uplift, International Journal for Numerical Methods in Engineering 65(8), 1197-1220, 2006.

[25] I. Planinc, M. Saje, B. Čas, On the local stability condition in the planar beam finite element, Structural Engineering and Mechanics 12(5), 507-526, 2001.

[26] B. Čas, Effect of flexible interface connection on stiffness and ultimate bearing capacity of composite beams, University of Ljubljana, Faculty of Civil and Geodetic Engineering, Internal Report, 1999.

[27] I. Planinc, S. Schnabl, M. Saje, J. Lopatič, B. Čas, Numerical and experimental analysis of timber composite beams with interlayer slip, Engineering Structures 30, 2959-2696, 2008.

[28] EN 338, Structural timber - Strength classes, European Committee for Standardization, EN 338:2003 E, 2003.

[29] R. Pischl, Mit kritischen betrachtungen and neuen vorschlägen zur bemessung nach theorie 1. und 2. ordnung, Holzbau: Technische Universität Graz, Fakultät für Bauingenierwesen, 1980. 
[30] W. J. McCutcheon, Stiffness of framing members with partial composite action, ASCE Journal of Structural Engineering 119(7), 1623-1637, 1986. 